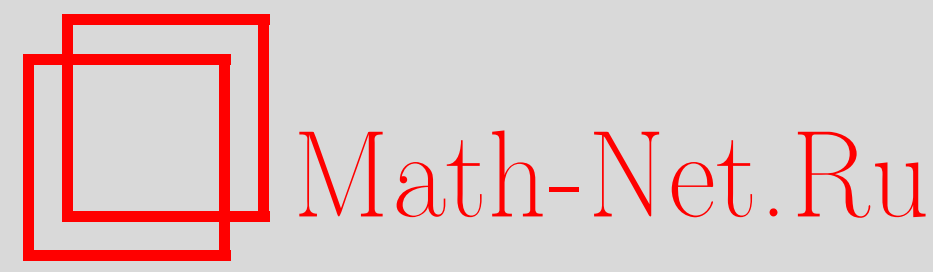

М. Д. Ковалёв, О распрямленных шарнирных конструкциях, Матем. сб., 2004, том 195, номер 6, 71-98

DOI: https://doi.org/10.4213/sm827

Использование Общероссийского математического портала Math-Net.Ru подразумевает, что вы прочитали и согласны с пользовательским соглашением

http: //www.mathnet.ru/rus/agreement

Параметры загрузки:

IP: 107.22 .136 .117

26 апреля 2023 г., 04:32:41 
УДК $514+531.8$

\author{
М. Д. Ковалев
}

\title{
О распрямленных шарнирных конструкциях
}

\begin{abstract}
До настоящего времени свойства наборов, состоящих из некоторых попарных расстояний между точками, брошенными в евклидово пространство, изучены мало. Эти свойства описьваются отображением, назьваемым автором рычажным (в англоязытных работах - "edge function", "rigidity mapping"), определяющим поведение реалњных конструкций из рычагов и шарниров. Наиболее простьм из нетривиальных оказывается случай рычажных отображений, отвечающих шарнирньм конструкциям (шарнирникам) в плоскости, все закрепленные шарниры которых лежат на одной прямой. В работе исследуются рычажные отображения и построены примеры таких распрямленных шарнирников с необычными свойствами.

Библиограффия: 13 названий.
\end{abstract}

\section{§1. Введение}

Содержание настоящей статьи непосредственно связано с теорией рычажношарнирных конструкций. Поэтому автор старался писать ее так, чтобы она была понятна возможно большему числу лиц, интересующихся этой теорией. Последнее привело, в частности, к включению в текст некоторых известных определений и утверждений.

Рассмотрим простейший пример закрепленной шарнирной схемы в плоскости (рис. 1) с одним свободным и двумя закрепленными в точках $(0,0)$ и $(1,0)$ шарнирами. Ему отвечает рычажное отображение $F: \mathbb{R}^{2} \rightarrow \mathscr{R}^{2}, F(x, y)=\left\{x^{2}+y^{2}\right.$, $\left.(x-1)^{2}+y^{2}\right\}$. Множество $B \subset \mathbb{R}^{2}$, на котором понижается ранг дифференциала этого отображения (множество вырождения), является прямой $y=0$. Через каждую ее точку $\mathbf{p}=\left(x_{0}, 0\right)$ можно провести прямую $L(\mathbf{p}): \mathbf{r}=\mathbf{p}+\operatorname{Ker} d F(\mathbf{p})$, в данном случае ее уравнение $x=x_{0}$. Образ $F(B)$ множества вырождения представляет собой гладкую кривую в $\mathscr{R}^{2}$, заданную параметрически уравнениями $d_{1}=x^{2}$, $d_{2}=(x-1)^{2},-\infty<x<+\infty$. Прямые $L(\mathbf{p})$ при отображении "перегибаются" и переходят в лучи $\mathscr{K}_{\mathbf{p}}$ с вершинами в точках кривой $F(B)$, имеющие направляющий вектор $(1,1)$. Множество $C$ значений рычажного отображения есть объединение всех лучей $\mathscr{K}_{\mathbf{p}}$. Касательньй конус $K_{\mathbf{p}}$ этого отображения в точке $\mathbf{p}$ (он состоит из лучей, касательных к образам всех гладких кривых, проходящих через р) является либо всей плоскостью $\mathscr{R}^{2}$ в случае, если $\mathbf{p} \notin B$, либо ее полуплоскостью в противном случае. Приведенное описание рычажного отображения во многом переносится и на случай распрямленных шарнирных схем в евклидовом пространстве $\mathbb{R}^{d}$,

Работа вьполнена при финансовой поддержке Российского фонда фундаментальных исследований (гранты № № 97-01-00266, 00-01-00619).

(C) М. Д. Ковалев 2004 


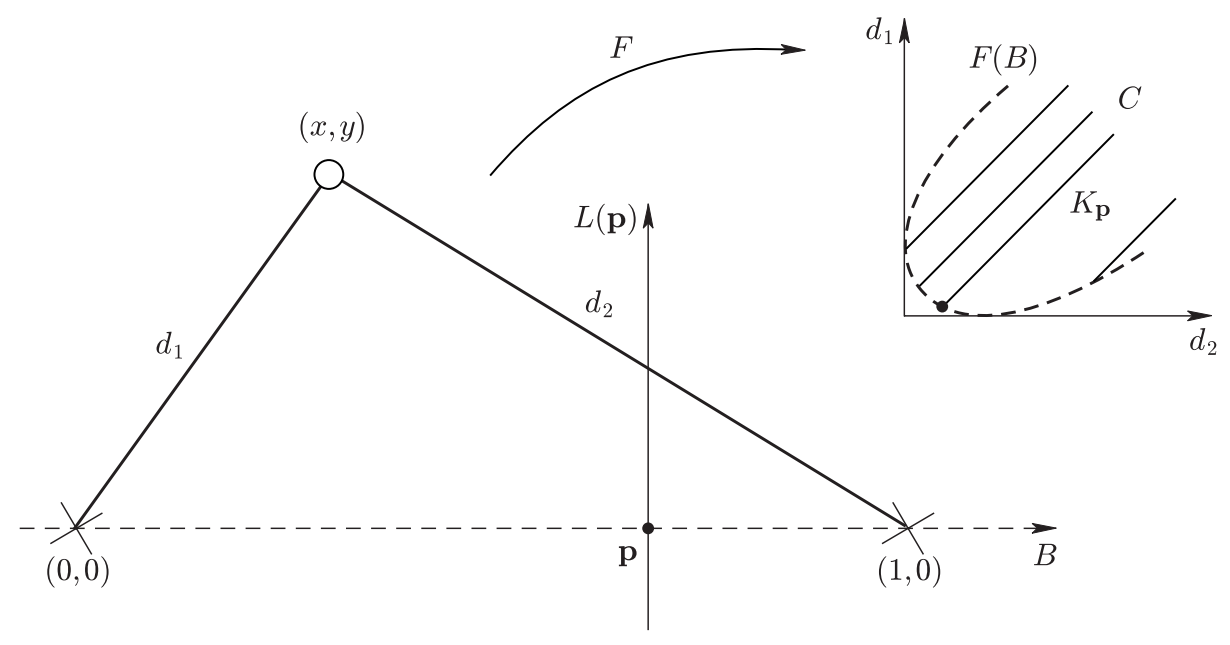

Рис. 1

$d \geqslant 2$. Хотя рычажное отображение часто возникало при изучении шарнирных конструкций (см. [1], [2]), его свойства еше мало исследованы.

В $\S 2$ приведены необходимые понятия геометрии шарнирных устройств, в частности закрепленной шарнирной схемы (ЗШС) как множества всех шарнирных конструкций данного комбинаторного строения с заданными положениями закрепленњых шарниров. Закрепленная в $\mathbb{R}^{d}$ шарнирная схема с $m$ свободными (незакрепленными) шарнирами определяет пространство-прообраз рычажного отображения $F: \mathbb{R}^{d m} \rightarrow \mathscr{R}^{r}$, каждая точка которого (отвечающая вполне определенной конструкции) называется шарнирником. Точки же пространства $\mathscr{R}^{r}$, где координатами являются квадраты длин рычагов шарнирника, называются кинематическими шарнирными схемами (КШС). Обсуждается понятие конуса рычажного отображения и его связь с устойчивостью шарнирника. Шарнирник устойчив, если при любой достаточно малой ошибке в длинах его рычагов можно будет собрать шарнирник, мало отличающийся от исходного. Очевидным необходимым условием устойчивости шарнирника $\mathbf{p} \in \mathbb{R}^{d m}$ является совпадение конуса рычажного отображения в точке $\mathbf{p}$ с пространством $\mathscr{R}^{r}$. Достаточное условие устойчивости шарнирника получено в $\S 4$ : КШС устойчива, если она является внутренней точкой множества $C=F\left(\mathbb{R}^{d m}\right)$. Отметим, что с устойчивостью связан ряд естественных, но пока открытых вопросов. Упомянем два из них. Первый-вопрос сушествования устойчивой КШС, которой бы отвечал лиш один шарнирник. Второй-вопрос существования устойчивой КШС, которой бы отвечали только лишш неустойчивые шарнирники (см. [3], [4]).

$\mathrm{B} \S 3$ приводятся некоторые свойства множества $C$ значений рычажного отображения и касательного конуса (контингенции) этого множества, обсуждается связь между контингенцией множества $C$ в точке $\mathbf{d} \in C$ и устойчивостью $\mathrm{K \amalg C} \mathbf{d}$. Внимание сосредотачивается на изостатических рычажных отображениях, т.е. отображениях с $d m=\max \operatorname{Rank} d F=r$. В изостатическом случае в точках, где ранг дифференциала отображения уменьшается лишь на единицу, конус отображения либо совпадает с $\mathscr{R}^{r}$, либо является полупространством этого пространства, либо 
$(r-1)$-мерной плоскостью [5]. Более сложным конус рычажного отображения может быть лишш в точках большего вырождения дифференциала. Этому условию удовлетворяют распрямленные шарнирники, т.е. такие, все шарниры которых лежат в некотором линейном подмногообразии рассматриваемого пространства.

Распрямленные шарнирники изучаются в $\S 4$. Справедлива простая теорема: на плоскости два распрямленных шарнирника с одной и той же КШС являются различньми положениями одного шарнирного механизма. Большая часть $\S 4$ посвящена выводу достаточного условия устойчивости шарнирника, используемого в $\S 5$. Это достаточное условие состоит в неравенстве нулю степени отображения-проектирования некоторого конуса вдоль касательного к рычажному отображению многообразия на дополнительное подпространство.

В $\S 5$ строится пример устойчивой распрямленной фермы (неизгибаемого шарнирника) в плоскости. Для этой фермы имеются изометричные фермы, естественно, не являюшиеся распрямленными.

В $\S 6$ описывается пример неустойчивой распрямленной фермы в плоскости, устойчивой по изменению длины каждого рычага в отдельности. Последняя ферма имеет ту же, не содержашую треугольных циклов, ЗШС, что и ферма из предыдушего примера. Построенный пример дает ответ на вопрос работы [3]. Потребовавшиеся для обоснования примеров достаточно сложные выкладки проводились на персональном компьютере с использованием пакета символьных вычислений Maple.

\section{§2. Рычажные отображения. Локальные свойства}

Определим, следуя [4], основной объект нашего исследования - рычажные отображения. Пусть $G_{1}\left(V_{1}, E_{1}\right)$ - абстрактный связный граф без петель и кратных ребер с вершинами $v_{1}, \ldots, v_{m} \in V_{1}$ и ребрами $v_{i} v_{j} \in E_{1}$. И пусть связньй граф $G(V, E)$ получается добавлением к $G_{1}\left(V_{1}, E_{1}\right)$ вершин $v_{m+1}, \ldots, v_{m+n}$, составляющих множество $V_{2}$, и некоторых ребер $v_{i} v_{j}, v_{i} \in V_{2}, v_{j} \in V_{1}$, составляющих множество $E_{2}$.

ОПРеДЕЛЕНИЕ 1. Графф $G(V, E), V=V_{1} \cup V_{2}, E=E_{1} \cup E_{2}$, назьвается шарнирной структурной схемой (IICC), ее вершины $v_{i} \in V_{2}-$ закрепленными, а $v_{j} \in V_{1}-$ свободнымм вершинами, причем, множества $V_{1}, E_{1}, V_{2}, E_{2}$ считаются непустыми.

Рассмотрим семейство $\mathbf{S}$ отображений ШСС в евклидово пространство $\mathbb{R}^{d}$, при котором ее закрепленные вершины переходят в фиксированные точки $p_{m+1}, p_{m+2}, \ldots, p_{m+n}$ плоскости, а ребра - в отрезки, соединяюшие образы инцидентных им вершин. Каждое такое отображение определяется заданием образов $p_{1}, p_{2}, \ldots, p_{m}$ свободных вершин и определяет иарнирник $\mathbf{p} \in \mathbb{R}^{d}$.

ОПРЕДЕЛЕнИЕ 2. Семейство отображений $\mathbf{S}$ назьвается закрепленной шарнирной схемой (ЗШС).

Следуя механическому смыслу, образы вершин при каждом отображении из $\mathbf{S}$ мы называем иарнирами, а образы ребер - рычагами. Свободным вершинам отвечают свободные, т.е. незакрепленные в $\mathbb{R}^{d}$ шарниры. Заметим, что не исключается совпадение любых двух (в том числе и смежных в графе $G$ ) шарниров как 
точек пространства $\mathbb{R}^{d}$. Пусть $|E|=r$, тогда ЗШС определяет отображение $F: \mathbb{R}^{d m} \rightarrow \mathscr{R}^{r}$, заданное формулами $d_{i j}=\left(p_{i}-p_{j}\right)^{2}, v_{i} v_{j} \in E$, где $d_{i j}$ есть квадрат длины рычага $p_{i} p_{j}$ шарнирника. Это отображение называется рычажнным.

ОПРЕДЕЛЕНИЕ 3 . Точка $\mathbf{d}=\left\{d_{i j}\right\} \in \mathscr{R}^{r}$ называется кинематической шарнирной схемой $($ КШC $)$, а точка $\mathbf{d} \in F\left(\mathbb{R}^{d m}\right)=C$ - существенной КШС.

Шарнирник $\mathbf{p}$ в $\mathbb{R}^{d}$ называют: жестким, если $\operatorname{Rank} d F(\mathbf{p})=d m ;$ неизгибаемымм (в инженерной терминологии - фермой), если он представляет собой одноточечную компоненту связности полного прообраза $F^{-1}(F(\mathbf{p}))$ своей КШС. Неизгибаемый шарнирник нельзя непрерывно двигать, не меняя длин его рычагов и положений закрепленных шарниров. Жесткий шарнирник неизгибаем, однако неизгибаемый шарнирник может быть нежестким. Таковым, например, является шарнирник, изображенный на рис. 1 , если все три его шарнира лежат на одной прямой. Изгибаемый шарнирник представляет собой определенное положение шарнирного механизма. С геометрической точки зрения шарнирный механизм можно отождествить с его конфигурационным пространством, т.е. с неодноточечной компонентой связности множества $F^{-1}(\mathbf{d})$. Шарнирники $\mathbf{p}$ и $\mathbf{p}^{\prime}$ называем изометричньими, если $F(\mathbf{p})=F\left(\mathbf{p}^{\prime}\right)$.

Если размерность множества $C$ сушественных КШС равна $r$, то отображение $F$ и соответствуюшая ЗШС называются правильныцми. Правильная ЗШС называется изостатической, если $d m=r$. Мы назьваем изостатическим и отвечающее ей рычажное отображение. Заметим, что для правильного рычажного отображения множество $B$ точек вырождения задается системой уравнений, выражаюших условие $\operatorname{Rank} d F(\mathbf{p})<r$, и представляет собой $(r-1)$-мерное алгебраическое множество в $\mathbb{R}^{d m}$. В случае изостатического рычажного отображения эта система превращается в одно полиномиальное уравнением $\operatorname{det} d F(\mathbf{p})=0$. Для правильной ЗШС несложно установить неограниченность множества $B$ [5].

Договоримся о некоторых понятиях, относяшихся к произвольному дифференцируемому отображению $F=\left\{f_{j}\right\}, 1 \leqslant j \leqslant r$. С точкой $p_{0}=\left\{x_{i}^{0}\right\} \in \mathbb{R}^{l}, 1 \leqslant i \leqslant l$, связано касательное $\kappa$ отображению $F$ в этой точке отображение, т.е. линейное отображение из $\mathbb{R}^{l}$ в $\mathscr{R}^{r}$, определенное формулами

$$
y_{j}=y_{j}^{0}+\sum_{j=1}^{l} \frac{\partial f_{j}}{\partial x_{i}}\left(x_{i}-x_{i}^{0}\right), \quad 1 \leqslant j \leqslant r, \quad\left\{y_{j}^{0}\right\}=F\left(p_{0}\right)=d_{0} .
$$

Матрицу этого отображения в стандартных (т.е. в тех, в которых задано отображение $F$ ) координатах мы обозначаем $d F\left(p_{0}\right)$. Ядерньм многообразием с иентром $p_{0}$ мы называем линейное многообразие $L\left(p_{0}\right) \subset \mathbb{R}^{l}$, состоящее из точек $p$, для которых векторы $p-p_{0}$ принадлежат ядру дифференциала отображения $F$ в точке $p_{0}$, т.е. $d F\left(p_{0}\right)\left(p-p_{0}\right)=0 \in \mathscr{R}^{r}$. Касательным многообразием $T\left(p_{0}\right) \subset \mathscr{R}^{r}$ отображения $F$ в точке $p_{0}$ мы называем линейное многообразие, состоящее из точек $d_{0}+d F\left(p_{0}\right)\left(p-p_{0}\right), p \in \mathbb{R}^{l}$. Имеет место простое утверждение [5].

ЛЕмма 1. Если ЗШС изостатическая, то каждая точка пространства параметров $\mathbb{R}^{d m}$ принадлежст некоторому ядерному многообразию рычажсного отображсения $F$. 
Квадратичным мы называем отображение $F: \mathbb{R}^{l} \rightarrow \mathscr{R}^{r}$ евклидовых пространств, заданное в декартовых координатных системах многочленами не вьше второй степени, среди которых обязательно есть хотя бы один многочлен второй степени. Квадратичное отображение называем однородным, если оно задано покоординатно квадратичными формами. Отображение называют собственным, если прообраз каждого компактного множества компактен. Справедлива следующая лемма [5].

Лемма 2. Однородное квадратичное отображсение $F$ является собственнымм тогда и только тогда, когда $F^{-1}(0)=0$.

Рычажное отображение квадратично и всегда является собственным [4]. Более того, однородная квадратичная часть рычажного отображения всегда является собственньм отображением [5]. Такие отображения мы называем вполне квадратичными.

Устойчивость шарнирников и кинематических схем подробно обсуждалась в работе [3]. Шарнирник $\mathbf{p}$ в $\mathbb{R}^{d}$ устойчия, если для любой его шаровой $\varepsilon$-окрестности $U(\mathbf{p}, \varepsilon) \subset \mathbb{R}^{d m}$ найдется $\delta$-окрестность $U(\mathbf{d}, \delta) \subset \mathscr{R}^{r}$ точки $\mathbf{d}=F(\mathbf{p})$, целиком лежащая в образе $F(U(\mathbf{p}, \varepsilon))$. Если достаточно мало изменить длины рычагов устойчивого шарнирника, то шарнирник с измененными длинами рычагов можно будет собрать таким образом, что все его шарниры окажутся близки к шарнирам исходного шарнирника. Устойчивый шарнирник непременно имеет правильную ЗШС. Назовем шарнирник $\mathbf{p}$ неособым, если $\operatorname{Rank} d F(\mathbf{p})=\max _{\mathbf{q} \in \mathbb{R}^{d m}} \operatorname{Rank} d F(\mathbf{q})=\eta$, и особым, если Rank $d F(\mathbf{p})<\eta$. Неособьй шарнирник с правильной ЗШС устойчив. В частности, всегда устойчива жесткая ферма с изостатической ЗШС. Как показывает пример плоского шарнирного четырехзвенника $p_{3} p_{1} p_{2} p_{4}$ с закрепленными шарнирами $p_{3}, p_{4}$ и прикрепленной к подвижному звену $p_{1} p_{2} p$ распрямленной двуповодковой группой $p_{1} p_{5} p_{2}$ (свободньй шарнир $p_{5}$ лежит на отрезке $\left.p_{1} p_{2}\right)$, сушествуют шарнирные механизмы, все положения которых суть неустойчивые шарнирники. Шарнирньй механизм этого примера является в некотором смысле исключительным, т.е. для всех его положений ранг дифференциала рычажного отображения меньше максимального. Назовем положение $\mathbf{p}_{0}$ шарнирного механизма с конфигурационным пространством $M$ исключительным, если каждый шарнирник $\mathbf{p} \in M$ из некоторой окрестности точки $\mathbf{p}_{0}$ является особьм, и неисключительным в противном случае. В работе [3] приведен пример шарнирного механизма с правильной ЗШС и переменным числом степеней свободы, обладающего исключительными положениями, представляющими собой неустойчивые шарнирники.

УТВЕРЖДЕНИЕ 1. Любое неисключительное положение иарнирного механизма с конфигуращионным пространством $M$ и правильной ЗШГС есть устойчивый шарнирник.

ДокАЗАТЕЛЬСтво. Если $\mathbf{p}_{0} \in M-$ правильньй шарнирник, то он, очевидно, устойчив. Пусть $\mathbf{p}_{0} \in M$ - неправильная точка отображения $F$, тогда в силу неисключительности точки $\mathbf{p}_{0}$ в произвольной ее шаровой $\varepsilon$-окрестности $U\left(\mathbf{p}_{0}, \varepsilon\right)$ найдется правильная точка $\mathbf{p} \in M$ и ее окрестность $U\left(\mathbf{p}, \varepsilon^{\prime}\right) \subset U\left(\mathbf{p}_{0}, \varepsilon\right)$. Но образ 
$F\left(U\left(\mathbf{p}, \varepsilon^{\prime}\right)\right)$ в силу правильности $\mathbf{p}$ покрьвает некоторую окрестность

$$
W(F(\mathbf{p}), \delta)=W\left(F\left(\mathbf{p}_{0}\right), \delta\right)
$$

с центром в точке $F\left(\mathbf{p}_{0}\right)$.

В $\S 5$ будет приведен пример, дающий отрицательный ответ на следующий вопрос работы [3]: любой ли неустойчивый шарнирник неустойчив по сколь угодно малому изменению длины одного лишь рычага? А именно, указан неустойчивый шарнирник $\mathbf{p}$, для которого $F(\mathbf{p})=\mathbf{d}=\left\{d_{i j}\right\}$ и $\forall \varepsilon>0$ и любого его рычага $v_{\mu} v_{\nu}$ найдется такое $\sigma>0$, что для любого числа $t, 0<|t|<\sigma, \mathrm{KWC} \mathbf{d}(t), d_{i j}(t)=d_{i j}$ при $(i, j) \neq(\mu, \nu)$ и $d_{\mu \nu}(t)=d_{\mu \nu}+t$, принадлежит множеству $F(U(\mathbf{p}, \varepsilon))$.

Для изучения устойчивости особых шарнирников и вообще свойств рычажного отображения "в малом" нам понадобится понятие (касательного) конуса отображения. Это понятие можно ввести для произвольного непрерывного отображения $F: \mathbb{R}^{l} \rightarrow \mathscr{R}^{r}$. Пусть при таком отображении $d=F(p)$. Рассмотрим всевозможные последовательности точек $p^{i} \in \mathbb{R}^{l}, i=1,2, \ldots$, сходящиеся к точке $p$, для которых $d_{i}=F\left(p^{i}\right) \neq d$. Таким последовательностям отвечают последовательности лучей $d d_{i} \in \mathscr{R}^{r}$. Множество $K_{p} \subset \mathscr{R}^{r}$ всех предельных для последовательностей $\left\{d d_{i}\right\}$ лучей мы называем касательным конусом или просто конусом отображсения $F$ в точке $p \in \mathbb{R}^{l}$. В случае полиномиального отображения $F$ конус $K_{p}$ можно построить следующим образом: рассмотреть всевозможные аналитические кривые в $\mathbb{R}^{l}$, проходящие через точку $p$. Множество лучей, касательных к образам этих кривых в точке $d=F(p)$, совпадает с конусом $K_{p}$. Более того, достаточно рассмотреть только кривые, имеющие полиномиальные параметризации, степени которых ограничены некоторой постоянной, зависящей лишш от отображения $F$.

Для устойчивости шарнирника $\mathbf{p}$, очевидньм образом, необходимо совпадение касательного конуса $K_{\mathbf{p}}$ с пространством $\mathscr{R}^{r}$. Однако не ясно - является ли это условие достаточньм для устойчивости шарнирника $\mathbf{p}$ ? Ответ на этот вопрос связан со следующим, также пока открытьм вопросом относительно общих квадратичных отображений. Собственному однородному квадратичному отображению $f: \mathbb{R}^{r} \rightarrow \mathscr{R}^{r}$ можно сопоставить отображение $f^{*}(x)=f(x) /|f(x)|$, переводящее проективное пространство $\mathbb{R} P^{r-1}$ в единичную сферу $\mathscr{S}^{r-1} \in \mathscr{R}^{r}$. Сушествует ли собственное однородное квадратичное отображение $f$ пространства $\mathbb{R}^{r}$ на все пространство $\mathscr{R}^{r}$, для которого бы $\operatorname{deg} f^{*}=0$ ? В случае нечетного $r$ степень $\operatorname{deg} f$ всегда равна 0, а степень $\operatorname{deg} f^{*}$ определена по модулю два и может не равняться 0 . В случае четного $r$ равенства $\operatorname{deg} f^{*}=0$ и $\operatorname{deg} f=0$ равносильны. При $r=2,3$ имеются собственные квадратичные отображения $f$ на все пространство $\mathscr{R}^{r}$, для которых $\operatorname{deg} f^{*} \neq 0$, а ответ на поставленный вопрос отрицателен [6].

Справедлива следуюшая теорема [5].

Tеорема 1. Пусть $F: \mathbb{R}^{r} \rightarrow \mathscr{R}^{r}-$ правильное квадратичное отображение, и пусть $\operatorname{dim} \operatorname{Ker} d F(p)=1$. Тогда конус $K_{p}$ отображения $F$ в точке $p$ представляет собой либо все пространство $\mathscr{R}^{r}$, либо его полупространство, либо $(r-1)$-мерную плоскость. Причем первые два варианта возможны, лишь когда р - изолированная точка множества $F^{-1} F(p)$, а последний имеет место в противном случае. 
В случае $\operatorname{dim} \operatorname{Ker} d F(p)>1$ дело обстоит сложнее. Однако иногда может помочь следуюшая теорема. Рассмотрим вешественно аналитическое в точке $p$ отображение $f$, и пусть $F$ есть квадратичная часть $f(p)+d f(p)+\frac{1}{2} d^{2} f(p)$ этого отображения. Допустим, для квадратичного отображения $F$ в точке $p$ ядерное многообразие $L(p)$ не пусто. Конус $\mathscr{K}_{p}=\mathscr{F}(L(p)-p), \mathscr{F}=\frac{1}{2} d^{2} f$, назовем составляющим конусом, а конус $Q_{p}=\mathscr{K}_{p}+T(p)$ - составным конусом отображения $f$ в точке $p$. Здесь знак сложения означает векторную сумму множеств в смысле Минковского. Имеет место очевидное включение $Q_{p} \subset K_{p}$. Если $\mathscr{K}_{p} \cap \operatorname{Im} d F(p)$ состоит из одной лишш вершины $0 \in \mathscr{R}^{r}$ конуса $\mathscr{K}_{p}$, то составной конус $Q_{p}$ представляет собой прямую сумму $\mathscr{K}_{p} \oplus \operatorname{Im} d F(p)$, перенесенную своей вершиной в точку $d=f(p)$. В этом случае между составным конусом и касательньм конусом отображения имеется следующая связь [5].

ТеОРема 2. Если квадратичная часть $F$ аналитического в точке $p$ отображения $f: \mathbb{R}^{l} \rightarrow \mathscr{R}^{r}$ вполне квадратична и $\mathscr{K}_{p} \cap \operatorname{Im} d F(p)=0$, то касательный конус $K_{p}$ отображения $f$ совпадает с его составным конусом $Q_{p}$.

\section{§ 3. Некоторые свойства множества существенных КШС}

О свойствах множества $C=F\left(\mathbb{R}^{d m}\right)$ известно немного. Это множество, как уже отмечалось, является полуалгебраическим связньм замкнутьм неограниченным и имеет в каждой своей точке одинаковую местную размерность, равную максимальному рангу матрицы $d F$. Установим еше несколько его свойств. Назовем точку $\mathbf{d}^{0} \in C$ разделяющей точкой множества $C$, если $C \backslash \mathbf{d}^{0}$ состоит из нескольких компонент $C_{1}, C_{2}, \ldots$ линейной связности. Справедлива следуюшая

Теорема 3. Если $\operatorname{dim} C>1$, то множество $C$ не имеет разделяющих точек.

ДокАЗАТЕЛЬСТво. Полный прообраз $M=F^{-1}\left(\mathbf{d}^{0}\right)$ представляет собой ограниченное множество в силу [4; лемма 1]. Это влечет принадлежность образов всех сфер $S^{d m-1}(r)$ достаточно большого радиуса $r \geqslant r_{0}$ с центрами в начале стандартной системы координат в $\mathbb{R}^{d m}$ какому-то одному из множеств $C_{i}$. Пусть, для определенности, множеству $C_{1}$. Тогда все остальные множества $C_{i}$ оказываются ограниченными, и их прообразы лежат внутри $S^{d m-1}\left(r_{0}\right)$. Возьмем точку $\mathbf{p}_{0} \in F^{-1}\left(C_{2}\right)$ и выпустим из нее лучи. Каждый такой луч пересечет множество $M$ до его пересечения со сферой $S^{d m-1}\left(r_{0}\right)$, поскольку его образ проходит через точку $\mathbf{d}^{0}$. Поскольку $M$ - алгебраическое множество, не совпадающее $\mathrm{c} \mathbb{R}^{d m}$, а его центральная проекция на сферу имеет размерность $d m-1$, то оно само с очевидностью имеет размерность $d m-1$. Это означает, что КШС $\mathbf{d}^{0}$ отвечает шарнирный механизм с $d m-1$ степенью свободы. Так как при $d=1$ не сушествует шарнирных механизмов, то остается разобрать лиш случай $d>1$. Покажем невозможность такого механизма, если число свободных шарниров больше одного. В самом деле, из двух различных свободных шарниров $p_{i}$ и $p_{j}$ выходят хотя бы два различных рыгага $p_{i} p_{l}$ и $p_{j} p_{s}$. Им отвечает пара уравнений $\left(p_{i}-p_{l}\right)^{2}=d_{i l}^{0}$, $\left(p_{j}-p_{s}\right)^{2}=d_{j s}^{0}$, где возможны случаи $l=j$ либо $s=i$. Пусть эта пара функций задает отображение $F^{*}$. Вследствие теоремы о неявной функции, если $\operatorname{dim} M=d m-1$ и точка $\mathbf{p} \in M$, то $\operatorname{Rank} d F^{*}(\mathbf{p}) \leqslant 1$. Однако множество $N \subset \mathbb{R}^{d m}$, 
на котором $\operatorname{Rank} d F^{*}(\mathbf{p}) \leqslant 1$, лежит в пересечении двух непараллельных плоскостей, а значит, имеет размерность, не превосходящую $d m-2$. Следовательно, $\operatorname{dim} M \leqslant d m-2$. Случай ЗШС с одним свободным шарниром не представляет затруднений. Теорема доказана.

Ответ на вопрос о принадлежности множества $C$ какой-либо $(r-1)$-мерной плоскости $P \subset \mathscr{R}^{r}$ дает следуюшая теорема. С механической точки зрения она описывает все закрепленные шарнирные схемы, для которых каждый шарнирник допускает одно и то же внутреннее напряжение [7].

Tеорема 4. Включение $C \subset P$ возможно тогда и только тогда, когда ЗНС имеет хотя бъ один свободный иарнир, например $v_{s}$, смежный не менее чем двум закрепленным шарнирам, например $v_{m+1}, v_{m+2}, \ldots, v_{m+k}, p a-$ диус-вектори $p_{m+1}, p_{m+2}, \ldots, p_{m+k}$ которых такови, что векторы $p_{m+2}-$ $p_{m+1}, \ldots, p_{m+k}-p_{m+1}$ линейно зависимы. При этом в уравнении $\sum a_{i j} d_{i j}=$ const плоскости Р коэффициенты $a_{i j}$ при квадратах длин рычагов, соединяющих свободные шарниры между собой, равны 0.

ДокаЗАТЕЛЬСтво. Подставив выражения $d_{i j}=\left(p_{i}-p_{j}\right)^{2}$ в уравнение плоскости $P$, получим квадратичный многочлен относительно переменных - координат свободных шарниров. Произведение первых координат $x_{i}^{1} x_{j}^{1} i$-го и $j$-го свободных шарниров содержится лишь в члене полученного уравнения, отвечаюшем рычагу $p_{i} p_{j}$. Поскольку справедливость уравнения не зависит от координат $x_{i}^{1}, x_{j}^{1}$ свободных шарниров, то коэффициент $a_{i j}$ равен 0 . Рассмотрим коэффициенты в уравнении плоскости $P$ при квадратах и первых степенях координат свободного шарниpa $v_{s}$, смежного закрепленньм шарнирам $v_{m+1}, v_{m+2}, \ldots, v_{m+k}$. Первый из них равен $\sum_{i=1}^{k} a_{s m+i}$, второй $-2 \sum_{i=1}^{k} a_{s m+i} x_{m+i}^{\nu}, 1 \leqslant \nu \leqslant d$. Выражая $a_{s m+1}$ из равенства $\sum_{i=1}^{k} a_{s ~ m+i}=0$ и подставляя в $\sum_{i=1}^{k} a_{s m+i} p_{m+i}=0$, получаем $\sum_{i=2}^{k} a_{s m+i}\left(p_{m+i}-p_{m+1}\right)=0$. Поскольку не все коэффициенты $a_{s} m+i$ равны нулю, последнее означает линейную зависимость векторов $p_{m+i}-p_{m+1}, 2 \leqslant i \leqslant k$. Обратно, предположив такую зависимость с коэффициентами $a_{s} m+i, 2 \leqslant i \leqslant k$, получим принадлежность множества $C$ плоскости $P$. Теорема доказана.

Для каждой ЗШС $G$ рассмотрим приведенную ЗШC $G^{*}$, получаемую из $G$ сведением всех закрепленных шарниров в один шарнир $p_{m+1}$, совпадающий с началом координат $O$ декартовой координатной системы в $\mathbb{R}^{d}$. Приведенной схеме $G^{*}$ отвечает рьчажное отображение $F^{*}$, являющееся однородной квадратичной частью рычажного отображения $F$, отвечаюшего схеме $G$. Множество $C^{*}$ сушественных КШС, отвечающих $G^{*}$, является конусом с вершиной в начале стандартной системы координат в $\mathscr{R}^{r}$, лежашим в неотрицательном ортанте $Q$. Мы будем называть $C^{*}$ предельным конусом исходного рычажного отображения $F$. В случае изостатической ЗШС $G$ размерность этого конуса меньше $r$. Например, закрепленной схеме рис. 2 отвечает приведенная ЗШС, у которой рычаги $p_{1} p_{3}$ и $p_{1} p_{4}$ совпадают. Соответствующий предельный конус $C^{*}$ лежит в трехмерном подпространстве $\mathscr{R}^{3}: d_{13}=d_{14}$ пространства $\mathscr{R}^{4}$. В этом подпространстве $\mathscr{R}^{3}$ можно пользоваться координатами $d_{12}, d_{13}, d_{25}$. В них трехмерный конус $C^{*}$ задается неравенствами $d_{12} \geqslant 0, d_{13} \geqslant 0, d_{25} \geqslant 0, \sqrt{d_{12}}+\sqrt{d_{13}} \geqslant \sqrt{d_{25}}, \sqrt{d_{12}}+\sqrt{d_{25}} \geqslant \sqrt{d_{13}}$, $\sqrt{d_{13}}+\sqrt{d_{25}} \geqslant \sqrt{d_{12}}$. 
Установим близость в определенном смысле множеств $C$ и $C^{*}$ для шарнирных схем в $\mathbb{R}^{d}$. Рассмотрим евклидово пространство $\mathbf{R}^{r}$ длин рычагов $r_{i j}=\sqrt{d_{i j}}$. Обозначим через $\mathbf{F}: \mathbb{R}^{d m} \rightarrow \mathbf{R}^{r}$ запись отображения $F$ в этих координатах, а через $\mathbf{F}^{*}$ - соответственно запись отображения $F^{*}$. Пусть $\mathbf{p}$ и $\mathbf{p}^{*}-$ шарнирники со схемами $G$ и $G^{*}$ соответственно, все одноименные свободные шарниры которых совпадают. Выберем координатную систему в $\mathbb{R}^{d}$ с началом $O=p_{m+1}$. Допустим, все закрепленные шарниры исходной ЗШС $G$ лежат в шаре радиуса $R$ с центром в точке $O$, и пусть $\rho(a, b)$ - евклидово расстояние между точками $a$ и $b$.

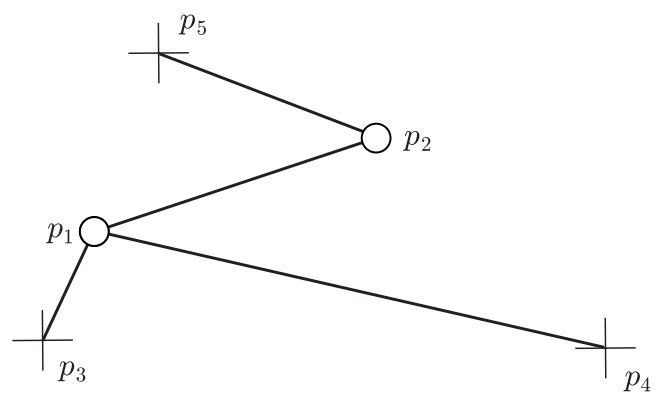

Рис. 2

ЛЕмма 3. Пусть $l$ - число рычагов, исходящих из всех за исключением $p_{m+1}$ закрепленных иарниров ЗШС $G$, а $D$ - длина наибольшего из этих рычагов в шарнирнике $\mathbf{p}$. Тогда при отображениях $\mathbf{F}$ и $F$ соответственно имеют место неравенства $\rho\left(\mathbf{F}(\mathbf{p}), \mathbf{F}^{*}\left(\mathbf{p}^{*}\right)\right) \leqslant R \sqrt{l}$ и $\rho\left(F(\mathbf{p}), F^{*}\left(\mathbf{p}^{*}\right)\right) \leqslant(2 D+R) R \sqrt{l}$.

ДоКАЗАТЕЛЬСТво. Используя неравенство треугольника, для свободного шарнира $p_{i}$ и закрепленного шарнира $p_{j}$ можно записать

$$
\rho\left(p_{i}^{*}, p_{j}^{*}\right)=\rho\left(p_{i}, O\right) \leqslant \rho\left(p_{i}, p_{j}\right)+\rho\left(p_{j}, O\right) \leqslant \rho\left(p_{i}, p_{j}\right)+R .
$$

Откуда $\left|r_{i j}-r_{i j}^{*}\right| \leqslant R$, если один из шарниров закреплен. Учитывая то, что $\left|r_{i j}-r_{i j}^{*}\right|=0$ для свободных шарниров $p_{i}$ и $p_{j}$, имеем

$$
\rho\left(\mathbf{F}(\mathbf{p}), \mathbf{F}^{*}\left(\mathbf{p}^{*}\right)\right)=\left(\sum_{i j}\left(r_{i j}-r_{i j}^{*}\right)^{2}\right)^{1 / 2} \leqslant R \sqrt{l} .
$$

Вторая оценка получается чуть сложнее. Из теоремы косинусов для треугольника $p_{i} p_{j} p_{j}^{*}$, в котором $p_{i}-$ свободный, а $p_{j}$ - закрепленньй шарниры, следует $d_{i j}-$ $d_{i j}^{*} \leqslant 2 R r_{i j}+R^{2}$. Откуда

$$
\rho\left(F(\mathbf{p}), F^{*}\left(\mathbf{p}^{*}\right)\right)=\left(\sum_{i j}\left(d_{i j}-d_{i j}^{*}\right)^{2}\right)^{1 / 2} \leqslant\left(2 R D+R^{2}\right) \sqrt{l}=(2 D+R) R \sqrt{l} .
$$

Пусть $\rho(x, A)=\inf _{y \in A} \rho(x, y)$ - расстояние от точки $x$ до множества $A$, тогда хаусдорфовым расстоянием $\rho(A, B)$ между множествами $A$ и $B$ называют 
$\sup _{x \in B, y \in A}\{\rho(x, A), \rho(y, B)\}$. Рассмотрим сечения $c C$ и $c C^{*}$ множеств $C$ и $C^{*}$ какой-либо плоскостью, отсекающей ограниченный кусок от неотрицательного ортанта стандартной координатной системы. Хаусдорфово расстояние между множествами $c C$ и $c C^{*}$ в силу оценки $\rho\left(c C, c C^{*}\right) \leqslant(2 D+R) R \sqrt{l}$ может быть не ограничено, поскольку величина $D$ не ограничена на множестве существенных КШС. Однако отношение $\rho\left(c C, c C^{*}\right)$ к $\operatorname{diam} c C$ стремится к 0 при заданном направлении секушей плоскости по мере того, как плоскость неограниченно отодвигается от начала координат.

Из доказанной леммы также следует оценка $\rho\left(c \mathbf{C}, c \mathbf{C}^{*}\right) \leqslant R \sqrt{l}$ хаусдорфовых расстояний для аналогично определенных множеств в пространстве $\mathbf{R}^{r}$ длин рычагов. Таким образом, расстояния между множествами $c \mathbf{C}$ и $c \mathbf{C}^{*}$ в пространстве $\mathbf{R}^{r}$ ограничены числом, не зависящим от секушей плоскости.

Сказанное позволяет сделать некоторые выводы о возможном строении множества $C$. Так для ЗШС шарнирника, изображенного на рис. 3 , множество $C^{*}$ является пятимерной конической поверхностью, лежащей в $\mathscr{R}^{6}$. В самом деле, конус $C^{*}$ представляет собой лежашую в ортанте $Q$ часть поверхности, которую можно задать уравнением $\Delta=0$, где $\Delta$ - определитель Кэли-Менгера [8]:

$$
\left|\begin{array}{ccccc}
0 & 1 & 1 & 1 & 1 \\
1 & 0 & d_{12} & d_{13} & d_{14} \\
1 & d_{21} & 0 & d_{23} & d_{24} \\
1 & d_{31} & d_{32} & 0 & d_{34} \\
1 & d_{41} & d_{42} & d_{43} & 0
\end{array}\right|
$$

Нетрудно проверить, что коническая поверхность $C^{*}$ разбивает пространство $\mathscr{R}^{6}$ на два связных куска. Отсюда вытекает наличие "полости" в множестве $C$ и отсутствие такого направления в $\mathscr{R}^{6}$, вдоль которого граница множества $C$ проектировалась бы взаимно однозначно на какое-либо пятимерное подпространство.

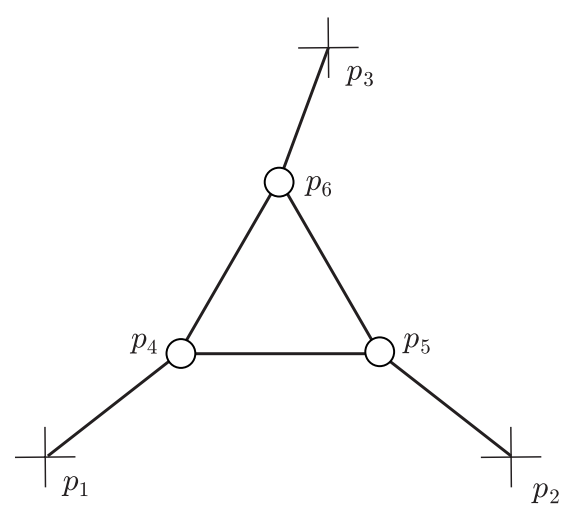

Рис. 3

Кинематическая шарнирная схема устойчива, если ей отвечает внутренняя точка множества $C$ сушественных КШС. Если КШС шарнирника р устойчива, то при любой достаточно малой ошибке в изготовлении рычагов шарнирник может 
быть собран, правда, некоторые свободные его шарниры возможно окажутся далеко от соответствующих шарниров шарнирника р. Для анализа устойчивости КШС вместо понятия касательного конуса отображения $F$ нужно рассмотреть понятие касательного конуса или контингенции множества $C=F\left(\mathbb{R}^{d m}\right)$. Конус $\mathbf{K}_{\mathbf{d}}$ множества $C$ в его предельной точке $\mathbf{d}$ представляет собой множество предельных лучей последовательностей лучей $\mathbf{d d}_{i}$, где $\left\{\mathbf{d}_{i}\right\}$ - всевозможные последовательности точек $\mathbf{d}_{i} \in C, \mathbf{d}_{i} \neq \mathbf{d}$, сходящиеся к точке $\mathbf{d}$. Необходимьм условием устойчивости КШС $\mathbf{d}$ является совпадение конуса $\mathbf{K}_{\mathbf{d}}$ с пространством $\mathscr{R}^{r}$. С другой стороны, автору неизвестны примеры, когда бы контингенция $\mathbf{K}_{\mathbf{d}}$ совпадала с пространством $\mathscr{R}^{r}$, а схема $\mathbf{d}$ была неустойчива. В случае рычажного отображения имеет место следующая связь контингенции с конусами отображения.

ЛЕмма 4. Справедливо равенство $\mathbf{K}_{\mathbf{d}}=\bigcup K_{\mathbf{p}}$, где обгединение берется по всем прообразам точки $\mathbf{d}$ при рычажсном отображении.

ДокаЗАТЕЛЬСтво. Включение $\mathbf{K}_{\mathbf{d}} \supset \bigcup K_{\mathbf{p}}$ очевидно. С другой стороњы, возьмем луч $\mathbf{d e} \in \mathbf{K}_{\mathbf{d}}$. Пусть он является предельным для последовательности лучей $\mathbf{d d}_{i}, \mathbf{d}_{i} \neq \mathbf{d}, \mathbf{d} \in C$. Из сходящейся к точке $\mathbf{d}$ последовательности точек $\mathbf{d}_{i}$ можно выбрать такую подпоследовательность $\mathbf{d}_{i_{j}}$, что последовательность точек $\mathbf{p}^{j} \in F^{-1}\left(\mathbf{d}_{i_{j}}\right)$ сходится к точке $\mathbf{p} \in \mathbb{R}^{d m}$. Это можно сделать вследствие ограниченности множества прообразов точек последовательности $\mathbf{d}_{i}$, вытекаюшей из собственности рычажного отображения. В силу непрерывности отображения $F$ имеем $\mathbf{p} \in F^{-1}(\mathbf{d})$. Таким образом, луч $\mathbf{d e} \in K_{\mathbf{p}}$ и $\mathbf{K}_{\mathbf{d}} \subset \bigcup K_{\mathbf{p}}$.

Хотя в простейших случаях и контингенция множества $C$, и конус рычажного отображения выпуклы, однако в более сложных случаях это не так. Уже для ЗШС шарнирника, изображенного на рис. 2, имеется КШС d, в которой контингенция множества $C$ невыпукла. В качестве таковой можно взять $\mathrm{K \amalg C} \mathbf{d}=F(\mathbf{p})$, где $\mathbf{p}-$ произвольный шарнирник, у которого $p_{1}=p_{5}$ и $p_{2} \neq p_{5}$. Такой шарнирник является положением шарнирного механизма с неподвижным шарниром $p_{1}$ и шарниpoм $p_{2}$, допускающим свободное вращение вокруг $p_{5}=p_{1}$. Пусть $\rho=\left|p_{2} p_{5}\right|>0$ и $W_{\rho} \in \mathbb{R}^{4}$ - конфигурационное пространство этого шарнирного механизма. Тогда в соответствии с теоремой 1 легко установить, что конус нашего рычажного отображения $F$ в точке $\mathbf{p}=(a, b, a+\rho \cos \varphi, b+\rho \sin \varphi) \in W_{\rho}$ является трехмерной плоскостью $T_{\varphi} \in \mathscr{R}^{4}$ с нормальным вектором $n_{\varphi}=(b,(1-a) \sin \varphi+b \cos \varphi$, $a \sin \varphi-b \cos \varphi,-b)$. В случае $\rho<b$ множество $F^{-1}(\mathbf{d})$ состоит только из шарнирников $\mathbf{p} \in W_{\rho}$. Вследствие леммы 4 контингенция множества $C$ в точке $\mathbf{d}$ в этом случае представляет собой объединение плоскостей семейства $T_{\varphi}, 0 \leqslant \varphi<2 \pi$. Рассматривая огибающую этого семейства плоскостей, нетрудно установить, что независимо от величины $\rho<b$ контингенции одинаковы и представляют собой цилиндр, построенный над внешней областью обычного эллиптического конуса, лежащего в некоторой трехмерной плоскости. Внешней областью конуса мы называем область пространства, ограниченную конической поверхностью и содержащую прямые, не проходящие через вершину конуса. Итак, даже если конус рычажного отображения для каждого из прообразов точки $\mathbf{d}$ является гиперплоскостью, контингенция множества $C$ в точке $\mathbf{d}$ может быть невыпуклой и иметь достаточно сложное строение. 


\section{§4. Распрямленные схемы и шарнирники}

Распрямленной мы называем закрепленную шарнирную схему в $\mathbb{R}^{d}$, линейная оболочка закрепленных шарниров которой является плоскостью $P$ размерности $k \geqslant 1$ меньшей $d$. Если и все свободные шарниры шарнирника с такой ЗШС лежат в плоскости $P$, то мы называем этот шарнирник распрямленныц. Распрямленные шарнирники в плоскости обладают следующим замечательным свойством.

ТЕОРемА 5. Два различных распрямленных изометричных шарнирника $\mathbf{p}$ $u \mathbf{p}^{\prime}$ в плоскости являются различными положсениями одного иарнирного механизма.

ДокАЗАТЕльство. Пусть все шарниры этих распрямленных шарнирников лежат на оси $O x$ декартовой координатной системы $O x y$ в плоскости, а $q_{i}=\left(x_{i}, 0\right)$ и $q_{i}^{\prime}=\left(x_{i}^{\prime}, 0\right)$ - положения $i$-го свободного шарнира соответственно шарнирников $\mathbf{p}$ и $\mathbf{p}^{\prime}$. Пусть $o_{i}=\left(x_{i}+x_{i}^{\prime}\right) / 2, O_{i}=\left(o_{i}, 0\right)$. Тогда если задать движение каждого свободного шарнира $p_{i}$ нашего шарнирника как равномерное врашение против часовой стрелки вокруг соответствуюшего центра $O_{i}$ из положения $q_{i}$ в положение $q_{i}^{\prime}$, то длины всех рычагов шарнирника при таком движении его шарниров будут неизменны. Действительно, если хотя бы один из шарниров $p_{i}, p_{j}$ неподвижен, то длина рычага $p_{i} p_{j}$ при таком движении, очевидно, неизменна. Рассмотрим теперь два подвижных шарнира $p_{i}, p_{j}$. Пусть векторы $q_{i} q_{j}$ и $q_{i}^{\prime} q_{j}^{\prime}$ сонаправлены. Тогда движение шарниров $p_{i}$ и $p_{j}$ описывается следующим образом:

$$
\begin{array}{ll}
x_{i}(t)=o_{i}+\left(x_{i}-o_{i}\right) \cos t, & y_{i}(t)=\left(x_{i}-o_{i}\right) \sin t \\
x_{j}(t)=o_{j}+\left(x_{j}-o_{j}\right) \cos t, & y_{j}(t)=\left(x_{j}-o_{j}\right) \sin t
\end{array}
$$

где $x_{i}-o_{i}=x_{j}-o_{j}$ и $0 \leqslant t \leqslant \pi$ независимо от знака разности $x_{i}-o_{i}$. В этом случае $d_{i j}(t)=\left(x_{i}(t)-x_{j}(t)\right)^{2}+\left(y_{i}(t)-y_{j}(t)\right)^{2}=\left(o_{i}-o_{j}\right)^{2}=\left(x_{i}-x_{j}\right)^{2}$. Если же векторы $q_{i} q_{j}$ и $q_{i}^{\prime} q_{j}^{\prime}$ противонаправлены, то имеем $o_{i}=o_{j}=o$ и

$$
\begin{array}{ll}
x_{i}(t)=o+\left(x_{i}-o\right) \cos t, & y_{i}(t)=\left(x_{i}-o\right) \sin t, \\
x_{j}(t)=o+\left(x_{j}-o\right) \cos t, & y_{j}(t)=\left(x_{j}-o\right) \sin t,
\end{array}
$$

где $0 \leqslant t \leqslant \pi$. В этом случае также $d_{i j}(t)=\left(x_{i}-x_{j}\right)^{2}$. Теорема доказана.

$\mathrm{B} \mathbb{R}^{3}$ аналогичное утверждение не имеет места (нетрудно построить примеры различных изометричных шарнирных ферм, лежаших в одной плоскости). Примеры нераспрямленных ферм в плоскости, изометричных распрямленной ферме, будут приведены в следуюшем параграфе.

Рассмотрим распрямленную ЗШС в $\mathbb{R}^{d}$. Будем считать, что в $\mathbb{R}^{d}$ выбрана декартова система координат $\left\{x_{j}\right\}$ с первыми $k$ осями, лежашими в плоскости $P$. Тогда множество $H$ распрямленных шарнирников представляет собой $\mathrm{km}$-мерное координатное подпространство $x_{j}^{i}=0,1 \leqslant j \leqslant k, 1 \leqslant i \leqslant m$, пространства параметров $\mathbb{R}^{d m}$. Пусть $L$ подпространство пространства $\mathbb{R}^{d m}$ ортогонально-дополнительное к $H$. Длины рычагов распрямленного шарнирника $\mathbf{p}$, очевидно, стационарны относительно скоростей свободных шарниров, ортогональных плоскости $P$, поэтому ядерное многообразие $L(\mathbf{p})$ содержит $(d-k) m$-мерную плоскость, 
параллельную подпространству $L$. Пусть $(\mathbf{x}, \mathbf{y})$, где $\mathbf{x} \in H$, a $\mathbf{y} \in L,-$ произвольный шарнирник. Тогда наше рычажное отображение $F$ можно представить в виде $F(\mathbf{x}, \mathbf{y})=F_{H}(\mathbf{x})+F_{L}(\mathbf{y})$, где $F_{H}$ и $F_{L}$ - сужения отображения $F$ на подпространства $H$ и $L$ соответственно. Тейлоровское разложение этого отображения в точке $\mathbf{p}=\left(\mathbf{x}_{\mathbf{p}}, \mathbf{0}\right)$, отвечаюшей распрямленному шарнирнику, в силу равенств $F_{L}(\mathbf{0})=0, d F_{L}(\mathbf{0}) \mathbf{y}=0$ вьглядит следующим образом:

$$
\begin{aligned}
F(\mathbf{x}, \mathbf{y}) & =F_{H}\left(\mathbf{x}_{\mathbf{p}}\right)+d F_{H}\left(\mathbf{x}_{\mathbf{p}}\right)\left(\mathbf{x}-\mathbf{x}_{\mathbf{p}}\right)+\frac{1}{2} d^{2} F_{H}\left(\mathbf{x}-\mathbf{x}_{\mathbf{p}}\right)+\frac{1}{2} d^{2} F_{L}(\mathbf{y}) \\
& =F_{H}(\mathbf{x})+\mathscr{F}_{L}(\mathbf{y})
\end{aligned}
$$

здесь $\mathscr{F} L-$ сужение однородной квадратичной части $\mathscr{F}$ нашего рычажного отображения на подпространство $L$. Назовем множество $M=F_{H}(H)=F(H)$ направляющим множсеством, а конус $\mathscr{K}=\mathscr{F}_{L}(L)=\mathscr{F}(L)$ - образующим конусом отображения $F$. Формула (1) означает, что множество $C$ сушественных КШС в случае распрямленной ЗШС представляет собой векторную сумму направляющего множества и образующего конуса отображения. Другими словами, $C$ можно представить как объединение конусов, полученных параллельным перенесением конуса $\mathscr{K}$ вершиной в точки множества $M$.

Если распрямленная ЗШС изостатическая, то размерность плоскости $P$ равняется $d-1$. Действительно, иначе бы возможно было вращение вокруг плоскости $P$ и не существовало бы нераспрямленных ферм с такой ЗШС, а множество ферм не было бы плотно в $\mathbb{R}^{d m}$, чего для изостатических схем не может быть. В случае изостатической распрямленной ЗШС множество $H$ распрямленных шарнирников представляет собой часть множества $B$ вырождения рычажного отображения. В этом случае ядерные многообразия с центрами на $H$ очевидным образом покрывают все пространство $\mathbb{R}^{d m}$. Заметим еще, что векторная сумма двух образующих конусов рычажного отображения в случае распрямленной ЗШС в плоскости представляет собой предельный конус $C^{*}$ этого отображения.

Если для распрямленного шарнирника $\mathbf{p}$ в $\mathbb{R}^{d}$ с изостатической ЗШС $\operatorname{Ker} d F(\mathbf{p})=L$, то составной конус $Q_{\mathbf{p}}=\mathscr{K}_{\mathbf{p}}+T(\mathbf{p})=\mathscr{K}+T(\mathbf{p})$. Если, кроме того, $\mathscr{K} \cap \operatorname{Im} d F(\mathbf{p})$ состоит из одной лишш вершины $0 \in \mathscr{R}^{r}$ конуса $\mathscr{K}$, то в силу теоремы 2 составной конус $Q_{\mathbf{p}}$ совпадает с касательным конусом $K_{\mathbf{p}}$. Следовательно, необходимым условием устойчивости шарнирника $\mathbf{p}$ в этом случае является равенство $\mathscr{K}+T(\mathbf{p})=\mathscr{R}^{r}$. Сейчас мы получим достаточное условие устойчивости такого распрямленного шарнирника р. Рассмотрим какое-либо $m$-мерное подпространство $W \subset \mathscr{R}^{r}$, дополнительное к его касательному многообразию $T(\mathbf{p})$. И пусть $\pi_{W}$ - отображение проектирования на подпространство $W$ вдоль многообразия $T(\mathbf{p})$, а отображение $\varphi: L \rightarrow W-$ наложение $\pi_{W} \mathscr{F}_{L}$. Тогда справедлива следуюшая теорема.

ТЕОРема 6. Если отображсние $\varphi$ собственное и его степень ненулевая, то иарнирник р устойчив.

Прежде чем переходить к доказательству этой теоремы, приведем необходимые понятия и утверждения. 
Для собственных отображений корректно определено понятие степени [9]. Более того, для доказательства этой теоремы нам понадобится понятие степени отображения или индекса ростка отображения в точке [10]. Рассмотрим аналитическое (в области своего определения) отображение $f: \mathbb{R}^{r} \rightarrow \mathscr{R}^{r}$ в окрестности точки $a$, являющейся изолированной точкой полного прообраза $f^{-1}(f(a))$. Пусть $S_{\varepsilon}=\partial U(a, \varepsilon) \subset \mathbb{R}^{r}$ - граничная сфера шаровой $\varepsilon$-окрестности точки $a$, а $S \in \mathscr{R}^{r}-$ сфера единичного радиуса с центром в точке $f(a)$.

Степенью $\operatorname{deg}_{a} f$ отображения $f$ в точке $а$ назьвается степень отображения

$$
\frac{f(x)-f(a)}{|f(x)-f(a)|}: S_{\varepsilon} \rightarrow S
$$

при достаточно малом $\varepsilon$. Если окрестность $U(a, \varepsilon)$ содержит лишш один прообраз точки $f(a)$, то степень последнего отображения не зависит от размера сферы $S_{\varepsilon}$. Степень отображения в точке $a$ равна с учетом знаков функциональных определителей числу прообразов правильной (это означает неравенство нулю функциональных определителей отображения во всех ее прообразах) точки $y$, достаточно близкой к $f(a)$, попавших в достаточно малую окрестность $U(a, \varepsilon)$ [10]. Степень отображения в невырожденной точке равна единице, в точке складки - нулю.

Имеет место утверждение.

ЛЕмма 5. Если полный прообраз $f^{-1}(d)$ собственного непрерывного отображения $f: \mathbb{R}^{r} \rightarrow \mathscr{R}^{r}$ состоит из конечного числа точек $p_{1}, \ldots, p_{k}$, то для любого $\varepsilon>0$ найдется такое $\delta>0$, что как только точка $d^{\prime} \in U(d, \delta)$, то полный ее прообраз $f^{-1}\left(d^{\prime}\right) \subset \bigcup_{i=1}^{k} U\left(p_{i}, \varepsilon\right)$.

ДокАЗАтЕльство. Действительно, допустим противное. Тогда для некоторого $\varepsilon>0$ найдется такая сходящаяся при $j \rightarrow \infty$ последовательность $d_{j} \rightarrow d$, что для каждого $j$ имеются точки $q_{j} \in f^{-1}\left(d_{j}\right)$, не принадлежашие множеству $M=\bigcup_{i=1}^{k} U\left(p_{i}, \varepsilon\right)$. В силу собственности отображения $f$ и ограниченности сходяшейся последовательности $\left\{d_{j}\right\}$ множество $\left\{q_{j}\right\}$ ограничено. Из ограниченной последовательности $\left\{q_{j}\right\}$ можно выбрать сходящуюся подпоследовательность $\left\{q_{j_{k}}\right\}$, пусть $q$ - ее предельная точка. Вследствие непрерывности нашего отображения $f(q)=\lim _{k \rightarrow \infty} f\left(q_{j_{k}}\right)=\lim _{k \rightarrow \infty} d_{j_{k}}=d$. Итак, точка $q$ должна совпадать с одной из точек $p_{1}, \ldots, p_{k}$, что противоречит выбору последовательности $\left\{q_{j}\right\}$.

Из леммы 5 очевидным образом вытекают следующие утверждения.

Лемма 6. Если полный прообраз $f^{-1}(d)$ аналитического собственного отображения $f$ состоит из точек $p_{1}, \ldots, p_{k}$, то имеет место равенство $\operatorname{deg} f=$ $\sum_{i=1}^{k} \operatorname{deg}_{p_{i}} f$.

СлЕДСТвИЕ. Если $\operatorname{deg}_{p} f \neq \operatorname{deg} f$ для собственного аналитического отображения $f$, то у точки $f(p)$ имеется кроме р еще хотя бъ один прообраз.

Следующее утверждение очевидно.

ЛЕмма 7. Пусть $f$ - аналитическое отображение, точка р является изолированной точкой полного прообраза $f^{-1}(f(p)) u \operatorname{deg}_{p} f \neq 0$. Тогда р устой- 
чива ${ }^{1}$ при отображсени $f$, т.е. для любой шаровой є-окрестности $U(p, \varepsilon)$ точки р найдется окрестность ее образа $U(f(p), \delta) \subset f(U(p, \varepsilon))$.

Пусть евклидово пространство $\mathbb{R}^{r}$ представлено в виде прямой суммы своих подпространств $X$ и $Y$. И пусть отображение $f^{\prime}: \mathbb{R}^{r} \rightarrow \mathscr{R}^{r}$ задается в стандартных декартовых системах координат следующим образом:

$$
f^{\prime}(x, y)=A x+g(y), \quad x \in X, \quad y \in Y
$$

где $A$ - невырожденная квадратная матрица, задающая линейньй гомеоморфизм подпространства $X$ на подпространство $T \in \mathscr{R}^{r}$, а $g(y)$ - однородное квадратичное отображение. Справедлива следующая лемма.

ЛЕмма 8. Если отображсение $g(y)$ собственное и $g(Y) \cap T=0$, то имеет место равенство $\operatorname{deg} f^{\prime}=\operatorname{deg}_{0} f^{\prime}$.

ДокАЗАтЕльство. Отображение $f^{\prime}(x, y)$, очевидно, собственно тогда и только тогда, когда собственно отображение $g(y)$. Условием собственности однородного квадратичного отображения $g(y)$ является равенство $g^{-1}(0)=0$. Поскольку $A x$ - линейный гомеоморфизм и $g(Y) \cap T=0$, то $f^{\prime-1}(0)=0$. Лемма доказана.

Прежде чем приступить к доказательству теоремы 6 , докажем лемму.

ЛЕмма 9. Если отображсение $\varphi$ собственное, то точка точка р изолированна в множестве $F^{-1}(F(\mathbf{p}))$.

ДоКАЗАТЕльство. Будем использовать координатные системы, приспособленные к точке р. Они получаются таким параллельным переносом стандартных декартовых систем в $\mathbb{R}^{d m}$ и в $\mathscr{R}^{r}$, чтобы их начала оказались соответственно в точках $\mathbf{p}$ и $F(\mathbf{p})$. В таких системах ядерное и касательное многообразия в точке $\mathbf{p}$ являются линейными подпространствами $L$ и $T$. Пусть $\boldsymbol{\xi}=\mathbf{x}-\mathbf{x}_{\mathbf{p}}$. Нам нужно доказать изолированность нулевого решения уравнения $F(\boldsymbol{\xi}, \mathbf{y})=0, \boldsymbol{\xi} \in H, \mathbf{y} \in L$, в этих координатах. Другими словами, нужно доказать существование окрестности начала координат $O \in \mathbb{R}^{d m}$, в которой эта точка - единственная точка прообраза $F^{-1}(0)$, $0 \in \mathscr{R}^{r}$. Проекция вектора $F(\boldsymbol{\xi}, \mathbf{y})$ на подпространство $W$ в силу разложения (1) записывается следующим образом:

$$
\pi_{W}\left(\mathscr{F}_{H}(\boldsymbol{\xi})+\mathscr{F}_{L}(\mathbf{y})\right)
$$

где $\mathscr{F}_{L}(\mathbf{y})=\mathscr{F}(\mathbf{0}, \mathbf{y})$ - сужение отображения $\mathscr{F}$ на подпространство $L$ и соответственно $\mathscr{F}_{H}(\boldsymbol{\xi})=\mathscr{F}(\boldsymbol{\xi}, \mathbf{0})$. Собственность однородного квадратичного отображения $\varphi$ вследствие леммы 2 и вполне квадратичности отображения $\mathscr{F} L(\mathbf{y})$ равносильна условию $\mathscr{K} \cap T=0$. В силу последнего условия и замкнутости образуюшего конуса $\mathscr{K}$ произвольный вектор конуса $\mathscr{K}$ образует с подпространством $T$ угол, больший некоторого угла $\nu, 0<\nu \leqslant \pi / 2$. В частности, это можно сказать

\footnotetext{
${ }^{1}$ Устойчивость точки при отображении - понятие, отличное от понятия устойчивости отображения в точке в теории особенностей дифференцируемых отображений. Первое из этих понятий представляет собой обобщение понятия устойчивости шарнирника. В общей топологии точку, устойчивую при отображении, назьвают точкой открытости этого отображения.
} 
про вектор $\mathscr{F}_{L}(\mathbf{y}) \in \mathscr{K}$. Поэтому из равенства $\pi_{W}\left(\mathscr{F}_{H}(\boldsymbol{\xi})+\mathscr{F}_{L}(\mathbf{y})\right)=0$ вытекает неравенство для длин векторов

$$
\frac{1}{\sin \nu}\left|\mathscr{F}_{H}(\boldsymbol{\xi})\right|>\left|\mathscr{F}_{L}(\mathbf{y})\right| .
$$

Пусть $\mathscr{A}$ - невырожденная квадратная матрица, задаюшая линейный гомеоморфизм $d F_{H}$ подпространства $H$, ортогонально-дополнительного к $L$, на $T$. Тогда проекция вектора $F(\boldsymbol{\xi}, \mathbf{y})$ на подпространство $T$ записывается следуюшим образом:

$$
\mathscr{A} \boldsymbol{\xi}+\pi_{T}\left(\mathscr{F}_{H}(\boldsymbol{\xi})+\mathscr{F}_{L}(\mathbf{y})\right),
$$

где $\pi_{T}$ - проекция на подпространство $T$ вдоль подпространства $W$. Так как $\mathscr{A}-$ линейный гомеоморфизм подпространства $H$ на $T$, то сушествует такая постоянная $a>0$, что

$$
|\mathscr{A} \boldsymbol{\xi}|>a|\boldsymbol{\xi}| .
$$

С другой стороны, для однородного квадратичного отображения $\mathscr{F}_{H}$ имеется такая постоянная $b>0$, что

$$
\left|\mathscr{F}_{H}(\boldsymbol{\xi})\right| \leqslant b|\boldsymbol{\xi}|^{2}
$$

(в качестве $b$ можно взять умноженную на $r$ наибольшую абсолютную величину собственных значений матриц квадратичных форм, задающих покоординатно отображение $\mathscr{F})$. Оценим величину проекции (3) учитывая то, что первый ее член имеет меньший чем остальные порядок малости:

$$
\begin{aligned}
\mid \mathscr{A} \boldsymbol{\xi} & +\pi_{T}\left(\mathscr{F}_{H}(\boldsymbol{\xi})+\mathscr{F}_{L}(\mathbf{y})\right)|\geqslant| \mathscr{A} \boldsymbol{\xi}|-| \pi_{T}\left(\mathscr{F}_{H}(\boldsymbol{\xi})+\mathscr{F}_{L}(\mathbf{y})\right) \mid \\
& \geqslant a|\boldsymbol{\xi}|-\frac{1}{\sin \lambda}\left|\mathscr{F}_{H}(\boldsymbol{\xi})+\mathscr{F}_{L}(\mathbf{y})\right| \geqslant a|\boldsymbol{\xi}|-\frac{1}{\sin \lambda}\left(\left|\mathscr{F}_{H}(\boldsymbol{\xi})\right|+\left|\mathscr{F}_{L}(\mathbf{y})\right|\right) \\
& \geqslant a|\boldsymbol{\xi}|-\frac{1}{\sin \lambda}\left(\left|\mathscr{F}_{H}(\boldsymbol{\xi})\right|+\frac{1}{\sin \nu}\left|\mathscr{F}_{H}(\boldsymbol{\xi})\right|\right) \geqslant a|\boldsymbol{\xi}|-\frac{1}{\sin \lambda}\left(b|\boldsymbol{\xi}|^{2}+\frac{b}{\sin \nu}|\boldsymbol{\xi}|^{2}\right) \\
& \geqslant|\boldsymbol{\xi}|\left(a-\frac{b|\boldsymbol{\xi}|}{\sin \lambda}\left(1+\frac{1}{\sin \nu}\right)\right),
\end{aligned}
$$

где $0<\lambda \leqslant \pi / 2-$ наименшший угол между векторами подпространств $W$ и $T$. Из этого неравенства вытекает, что при условии

$$
|\boldsymbol{\xi}|<\varepsilon=\frac{a \sin \lambda}{b}\left(1+\frac{1}{\sin \nu}\right)^{-1}
$$

имеется лишь одно, а именно нулевое, решение уравнения $F(\boldsymbol{\xi}, \mathbf{y})=0$. Лемма доказана.

Заметим теперь, что справедливость теоремы 6 вытекает с учетом леммы 7 из следуюшей леммы.

ЛЕмма 10. В условиях теоремы 6 справедливо равенство

$$
\left|\operatorname{deg}_{\mathbf{p}} F\right|=|\operatorname{deg} \varphi|
$$


ДоказАТЕЛЬСтво. Рассмотрим отображение $F^{\prime}$, приближаюшее рычажное отображение $F$ в случае распрямленной ЗШС. Геометрически отображение $F^{\prime}$ получается из $F$, если заменить в векторной сумме (1) направляющее множество его касательным в точке $\mathbf{p}$ многообразием. Ввиду (1) отображение $F^{\prime}$ задается в приспособленных к точке $\mathbf{p}$ системах координат следующей формулой:

$$
F^{\prime}(\boldsymbol{\xi}, \mathbf{y})=\mathscr{A} \boldsymbol{\xi}+\mathscr{F}_{L}(\mathbf{y}), \quad \boldsymbol{\xi} \in H, \quad \mathbf{y} \in L .
$$

Сначала мы установим равенство $\left|\operatorname{deg} F^{\prime}\right|=|\operatorname{deg} \varphi|$, а затем равенство $\operatorname{deg}_{O} F=$ $\operatorname{deg} F^{\prime}$ (мы считаем отображение $F$ записанным в приспособленной к точке $\mathbf{p}$ системе координат, где р совпадает с началом координат $O$ ), что и завершит доказательство теоремы. Пусть $\vartheta(\mathbf{y})=\pi_{T}(\mathscr{F} L(\mathbf{y}))$, тогда составляющие отображения $F^{\prime}$ по подпространствам $W$ и $T$ соответственно имеют вид $\varphi(\mathbf{y})$ и $\mathscr{A} \boldsymbol{\xi}+\vartheta(\mathbf{y})$. Для доказательства равенства $\left|\operatorname{deg} F^{\prime}\right|=|\operatorname{deg} \varphi|$ достаточно установить взаимно однозначное соответствие между множествами решений системы уравнений $\varphi(\mathbf{y})=w$ и системы

$$
\left\{\begin{array}{l}
\varphi(\mathbf{y})=w \\
\mathscr{A} \boldsymbol{\xi}+\vartheta(\mathbf{y})=t
\end{array}\right.
$$

при одинаковых значениях $w \in W$ и произвольном $t \in T$ и доказать одинаковость либо противоположность знаков функциональных определителей для каждой пары соответствуюших друг другу решений. Поскольку $\boldsymbol{\xi}$ однозначно определяется из второй системы как функция у равенством $\boldsymbol{\xi}=\mathscr{A}^{-1}(t-\vartheta(\mathbf{y}))$, то каждому решению $\mathbf{y}$ первой системы отвечает единственное решение $(\boldsymbol{\xi}, \mathbf{y})$ второй системы, каков бы ни был вектор $t \in T$. И наоборот, поскольку не сушествует двух различных решений второй системы, отличаюшихся лишш значениями $\boldsymbol{\xi}$, то каждому решению $(\boldsymbol{\xi}, \mathbf{y})$ второй системы с фиксированньм $t$ отвечает единственное решение у первой. Функциональная матрица $d F^{\prime}$, если в первых $m$ ее строках стоят производные координатных функций из подпространства $W$, а в первых $m$ столбцах - производные по координатам из $L$, состоит из четырех блоков. Квадратный блок, расположенный в ее левом верхнем углу, представляет собой матрицу $d \varphi$. Квадратный блок в правом нижнем углу - матрицу $\mathscr{A}$. Прямоугольньй блок, расположенный над блоком $\mathscr{A}$, является нулевой матрицей. Раскладывая определитель матрицы $d F^{\prime}(\boldsymbol{\xi}, \mathbf{y})$ по первым его $m$ строкам, получаем равенство $\operatorname{det} d F^{\prime}(\boldsymbol{\xi}, \mathbf{y})=\operatorname{det} d \varphi(\mathbf{y}) \operatorname{det} \mathscr{A}$. Таким образом, $\operatorname{deg} F^{\prime}$ и $\operatorname{deg} \varphi$ совпадают, если $\operatorname{det} \mathscr{A}>0$, и различаются знаком, если $\operatorname{det} \mathscr{A}<0$.

Докажем теперь равенство $\operatorname{deg}_{O} F=\operatorname{deg} F^{\prime}$. Пусть однородные квадратичные отображения $\phi(\boldsymbol{\xi})$ и $\theta(\boldsymbol{\xi})$ определены формулами $\phi(\boldsymbol{\xi})=\pi_{W} \mathscr{F}_{H}(\boldsymbol{\xi})$ и $\theta(\boldsymbol{\xi})=$ $\pi_{T} \mathscr{F}_{H}(\boldsymbol{\xi})$. Тогда отображение $F$ задается формулами

$$
\begin{aligned}
& \varphi(\mathbf{y})+\phi(\boldsymbol{\xi})=w, \\
& \mathscr{A} \boldsymbol{\xi}+\vartheta(\mathbf{y})+\theta(\boldsymbol{\xi})=t .
\end{aligned}
$$

В силу леммы 8 для отображения $F^{\prime}$ справедливо равенство $\operatorname{deg}_{O} F^{\prime}=\operatorname{deg} F^{\prime}$. Поэтому нам остается установить равенство $\operatorname{deg}_{O} F=\operatorname{deg}_{O} F^{\prime}$. Для этого вследствие инвариантности степени отображения при гомотопии [9] достаточно установить для произвольно малого $\varepsilon>0$ наличие шаровой $\delta$-окрестности $U(O, \delta)$ начала $O$ приспособленной к р системы координат, в которой будет выполнено неравенство

$$
\left|F(\boldsymbol{\xi}, \mathbf{y})-F^{\prime}(\boldsymbol{\xi}, \mathbf{y})\right|<\varepsilon\left|F^{\prime}(\boldsymbol{\xi}, \mathbf{y})\right| .
$$


Действительно, учитывая то, что наименьший угол между векторами из $T$ и $\mathscr{K}$ равен $\nu$, и используя $(*)$, запишем

$$
\left|F^{\prime}(\boldsymbol{\xi}, \mathbf{y})\right|=\left|\mathscr{A} \boldsymbol{\xi}+\mathscr{F}_{L}(\mathbf{y})\right| \geqslant|\mathscr{A} \boldsymbol{\xi}| \sin \nu>a|\boldsymbol{\xi}| \sin \nu .
$$

С другой стороны, с учетом $(* *)$ имеем

$$
\left|F(\boldsymbol{\xi}, \mathbf{y})-F^{\prime}(\boldsymbol{\xi}, \mathbf{y})\right|=|\phi(\boldsymbol{\xi})+\theta(\boldsymbol{\xi})|=\left|\mathscr{F}_{H}(\boldsymbol{\xi})\right| \leqslant b|\boldsymbol{\xi}|^{2} .
$$

Из последних двух неравенств следует достаточность для выполнения неравенства (6) условия $\delta \leqslant(a \sin \nu / b) \varepsilon$. Итак, лемма 10 , а вместе с ней и теорема 6 доказаны.

Распрямленные шарнирники с изостатической ЗШС и не слишком малым числом шарниров сильно вырождены, и поэтому для них можно ожидать существования касательных конусов рычажного отображения с необычньми свойствами. Однако в следующем простом случае конус рычажного отображения всегда содержится в полупространстве.

Теорема 7. Пусть ЗШС содержит треугольный иикл $\Delta=p_{a} p_{b} p_{c}$ либо шарниры $p_{a}$ и $p_{c}$ закрепленнье, а свободный шарнир $p_{b}$ соединен с ними двумя рычагами. Тогда если в шарнирнике $\mathbf{p}$ шарниры $p_{a}, p_{b}, p_{c}$ лежат на одной прямой, то конус $K_{\mathbf{p}}$ рычажного отображсения лежст в полупространстве пространства $\mathscr{R}^{r}$.

ДокАЗАТЕльство. Рассмотрим случай цикла, квадраты длин рычагов которого равны $d_{a b}, d_{b c}, d_{c a}$. Если среди этих трех чисел более одного наибольшего, то одно из них равно нулю, и точка $\mathbf{d}=F(\mathbf{p})$ лежит на границе ортанта $Q$. В силу вьпуклости $Q$ конус $K_{\mathbf{p}}$ заключен в некотором полупространстве. В противном случае пусть $d_{a b}$ единственная наибольшая из этих трех величин. Тогда множество $C$ сушественных КШС принадлежит множеству $D \subset Q$, на котором цикл $\Delta$ сушествует. Но точка $\mathbf{d}$ в этом случае является точкой гладкости границы множества $D$. Действительно, в окрестности точки $\mathbf{d}$ множество $D$ задается неравенством $\sqrt{d_{a b}} \leqslant \sqrt{d_{b c}}+\sqrt{d_{c a}}$. Так как касательньй конус множества $D$ в точке $\mathbf{d}$ есть полупространство, то конус $K_{\mathbf{p}}$ лежит в этом полупространстве. Случай закрепленных шарниров разбирается аналогично.

\section{§5. Пример устойчивой распрямленной фермы}

Теорема 5 влечет естественный вопрос о сушествовании в плоскости изометричных друг другу распрямленной и нераспрямленной шарнирных ферм. Здесь приведен пример, дающий положительный ответ на этот вопрос. Одновременно он является примером устойчивой распрямленной шарнирной фермы, отвечаюшим на вопрос работы [11].

Учитьвая теорему 7 , нужно искать такой пример среди шарнирников со схемой, не содержашей треугольных циклов. Распрямленная ЗШС нашего примера - одна из простейших плоских изостатических схем такого рода - изображена на рис. 4 . На рис. 4 а) изображена ферма, доставляюшая наш пример. Она представляет собой распрямленный шарнирник $\mathbf{p}$ в плоскости, имеющий четыре закрепленных шарнира: $p_{5}=(0,0), p_{6}=(1,0), p_{7}=\left(v_{7}, 0\right), p_{8}=\left(v_{8}, 0\right)$. Причем $v_{7}=-1, v_{8}=2$, 
однако для общности дальнейших подсчетов мы не будем выполнять подстановки сразу. Четыре его свободных шарнира лежат на прямой $P: y=0$ в точках $x_{1}=3$, $x_{3}=2, x_{2}=4, x_{4}=6$. Рьчаги по порядку таковы: $p_{5} p_{1}, p_{6} p_{2}, p_{7} p_{3}, p_{8} p_{4}, p_{1} p_{2}$, $p_{1} p_{4}, p_{2} p_{3}, p_{3} p_{4}$. Напишем матрицу дифференциала соответствуюшего рычажного отображения, вьписывая в первых четырех столбцах ее элементы, отвечающие ненулевым координатам шарниров шарнирника $\mathbf{p}$ :

$$
2\left(\begin{array}{cccccccc}
x_{1} & 0 & 0 & 0 & 0 & 0 & 0 & 0 \\
0 & x_{2}-1 & 0 & 0 & 0 & 0 & 0 & 0 \\
0 & 0 & x_{3}-v_{7} & 0 & 0 & 0 & 0 & 0 \\
0 & 0 & 0 & x_{4}-v_{8} & 0 & 0 & 0 & 0 \\
x_{1}-x_{2} & x_{2}-x_{1} & 0 & 0 & 0 & 0 & 0 & 0 \\
x_{1}-x_{4} & 0 & 0 & x_{4}-x_{1} & 0 & 0 & 0 & 0 \\
0 & x_{2}-x_{3} & x_{3}-x_{2} & 0 & 0 & 0 & 0 & 0 \\
0 & 0 & x_{3}-x_{4} & x_{4}-x_{3} & 0 & 0 & 0 & 0
\end{array}\right) .
$$

Касательное многообразие $T(\mathbf{p})=d F(\mathbf{p}) \cdot H \subset \mathscr{R}^{8}$ нашего рычажного отображения $F$ в точке р натянуто на четыре вектора $e_{1}, e_{2}, e_{3}, e_{4}$, координатные столбцы которых в стандартном базисе в $\mathscr{R}^{8}$ являются первыми четырымя столбцами матрицы $d F$, взятыми по порядку и умноженными на $\frac{1}{2}$. Если свободные шарниры не совпадают с закрепленными, как это имеет место для шарнирника $\mathbf{p}$, то эти четыре вектора линейно независимы. Возьмем четыре вектора $e_{5}=(0,0,0,0,1,0,0,0)$, $e_{6}=(0,0,0,0,0,1,0,0), e_{7}=(0,0,0,0,0,0,1,0), e_{8}=(0,0,0,0,0,0,0,1)$, дополняющие первую четверку до базиса $\mathscr{E}$ в $\mathscr{R}^{8}$. Матрица $M$ перехода от координат в стандартном базисе в $\mathscr{R}^{8}$ к координатам в базисе $\mathscr{E}$ обратна к матрице, столбцами которой являются векторы $e_{i}, 1 \leqslant i \leqslant 8$. Вычисления дают

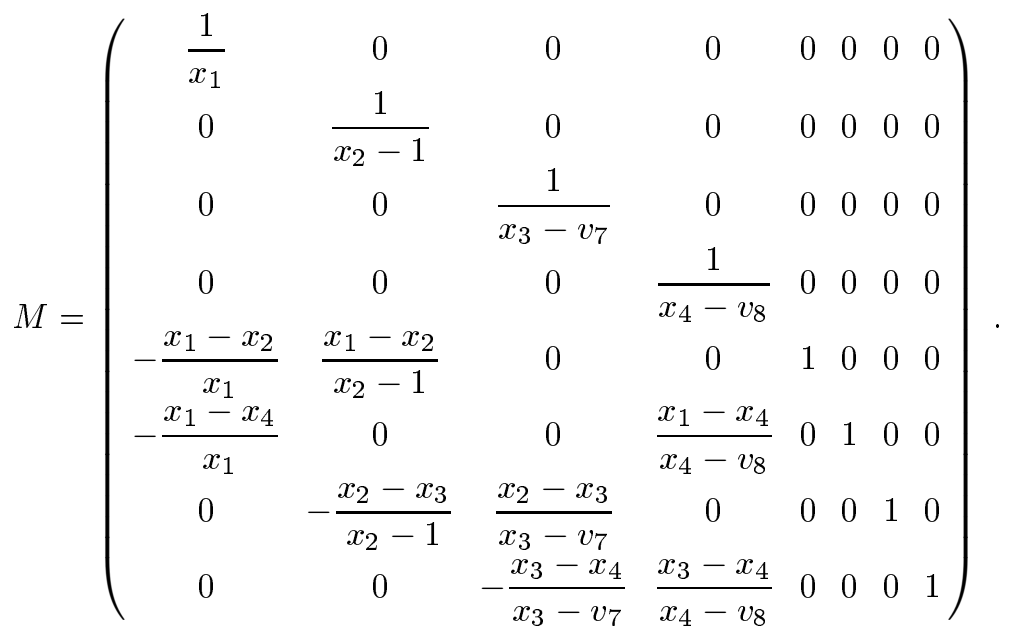

Ядерное многообразие касательного отображения в точке $\mathbf{p}$ состоит из векторов, которые имеют нулевые абсциссы в $\mathbb{R}^{2}$ и ординаты которых могут принимать произвольные вещественные значения: $\left(y_{1}, y_{2}, y_{3}, y_{4}\right)=\mathbf{y} \in L(\mathbf{p})=L$. Его образ образующий конус нашего рычажного отображения - задается в стандартном базисе следующим образом:

$$
\mathscr{K}=\left(y_{1}^{2}, y_{2}^{2}, y_{3}^{2}, y_{4}^{2},\left(y_{1}-y_{2}\right)^{2},\left(y_{1}-y_{4}\right)^{2},\left(y_{2}-y_{3}\right)^{2},\left(y_{3}-y_{4}\right)^{2}\right) .
$$




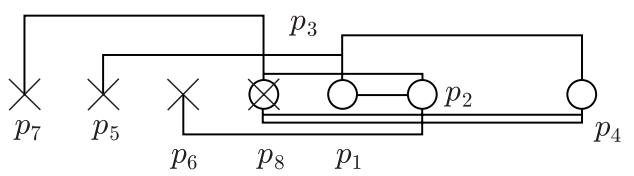

a)

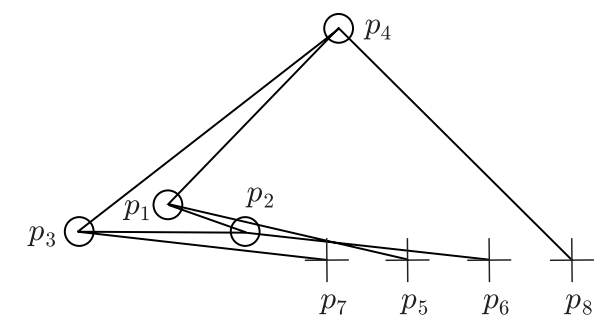

b)

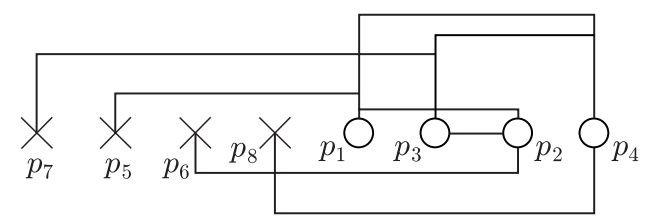

c)

Рис. 4

Координаты вектора в базисе $\mathscr{E}$ получаются умножением матрицы $M$ на координатный столбец этого вектора в базисе стандартной системы координат в $\mathscr{R}^{8}$. Причем последние четыре координаты откладьваются по одним и тем же осям в стандартном базисе и в базисе $\mathscr{E}$. Умножая матрицу $M$ на векторы образуюшего конуса $\mathscr{K}$ и выбирая последние четыре координаты получаюшихся векторов, мы найдем проекцию этого образуюшего конуса на четырехмерное координатное подпространство $\mathscr{R}^{4}$, порожденное четырьмя последними векторами как базиса $\mathscr{E}$, так и стандартного базиса в $\mathscr{R}^{8}$. Это будет конус $\pi \mathscr{K}$ :

$$
\begin{aligned}
\pi \mathscr{K}=( & -\frac{\left(x_{1}-x_{2}\right) y_{1}^{2}}{x_{1}}+\frac{\left(x_{1}-x_{2}\right) y_{2}^{2}}{x_{2}-1}+\left(y_{1}-y_{2}\right)^{2} \\
& -\frac{\left(x_{1}-x_{4}\right) y_{1}^{2}}{x_{1}}+\frac{\left(x_{1}-x_{4}\right) y_{4}^{2}}{x_{4}-v_{8}}+\left(y_{1}-y_{4}\right)^{2} \\
& -\frac{\left(x_{2}-x_{3}\right) y_{2}^{2}}{x_{2}-1}+\frac{\left(x_{2}-x_{3}\right) y_{3}^{2}}{x_{3}-v_{7}}+\left(y_{2}-y_{3}\right)^{2} \\
& \left.-\frac{\left(x_{3}-x_{4}\right) y_{3}^{2}}{x_{3}-v_{7}}+\frac{\left(x_{3}-x_{4}\right) y_{4}^{2}}{x_{4}-v_{8}}+\left(y_{3}-y_{4}\right)^{2}\right) \\
\left(y_{1}, y_{2}, y_{3}, y_{4}\right) \in L &
\end{aligned}
$$

Подставляя сюда координаты шарниров шарнирника $\mathbf{p}$, получаем

$$
\pi \mathscr{K}=\left(\frac{4}{3} y_{1}^{2}+\frac{2}{3} y_{2}^{2}-2 y_{1} y_{2}, 2 y_{1}^{2}+\frac{1}{4} y_{4}^{2}-2 y_{1} y_{4}, \frac{1}{3} y_{2}^{2}+\frac{5}{3} y_{3}^{2}-2 y_{2} y_{3}, \frac{7}{3} y_{3}^{2}-2 y_{3} y_{4}\right) \text {. }
$$


Рассмотрим квадратичное отображение $\varphi: L \rightarrow \mathscr{R}^{4}$, определенное покоординатно формулами (8). Система уравнений с левьми частями из (8) и нулевыми правыми частями имеет тривиальное нулевое решение. Чтобы установить собственность отображения $\varphi$, достаточно в силу леммы 2 доказать единственность этого тривиального решения. Допустим сушествование у нее ненулевого решения, тогда вследствие однородности система имеет решение $\mathbf{y}=\left(y_{1}, y_{2}, y_{3}, y_{4}\right)$ либо с $y_{4}=1$, либо с $y_{4}=0$. В первом случае получаем следуюшую систему уравнений для определения $y_{1}, y_{2}, y_{3}$ :

$$
\left\{\begin{array}{l}
\frac{4}{3} y_{1}^{2}+\frac{2}{3} y_{2}^{2}-2 y_{1} y_{2}=0 \\
2 y_{1}^{2}+\frac{1}{4}-2 y_{1}=0 \\
\frac{1}{3} y_{2}^{2}+\frac{5}{3} y_{3}^{2}-2 y_{2} y_{3}=0 \\
\frac{7}{3} y_{3}^{2}-2 y_{3}=0
\end{array}\right.
$$

Из 4-го и 2-го уравнений системы (9) найдем по два возможных значения $y_{3}$ и $y_{1}$ :

$$
\begin{aligned}
& y_{3}=0, \frac{6}{7} \\
& y_{1}=\frac{1}{2}+\frac{1}{4} \sqrt{2}, \frac{1}{2}-\frac{1}{4} \sqrt{2} .
\end{aligned}
$$

Подставляя все возможные пары $\left(y_{1}, y_{3}\right)$ в 1-е и 3-е уравнения системы (9), получаем четыре системы из двух квадратных уравнений относительно $y_{2}$. Все четыре системы, как легко проверить, не имеют вешественных решений. Следовательно, система (9) несовместна и не сушествует вешественного решения у исходной системы с $y_{4} \neq 0$.

Из предположения $y_{4}=0$ следуют равенства $y_{3}=0, y_{2}=0$ и $y_{1}=0$. Таким образом, система имеет только тривиальное решение. Чтобы теперь применить теорему 6 , необходимо найти степень отображения $\varphi$. Для этого с помощью пакета Maple найдены в точном виде (как выражения через корни многочленов от одного переменного) все 16 решений системы уравнений с левыми частями - координатами вектора из (8) и правыми частями - координатами вектора $(0,0,1,0)$. Эти 16 решений естественньм образом распадаются на 8 пар, различаюшихся знаком. Поскольку точные выражения решений весьма громоздки, мы приведем лишь их приближенные значения - по одному для каждой из 8 пар:

$\begin{array}{cccc}y_{1} & y_{2} & y_{3} & y_{4} \\ 0.8660 & 1.7320 & 0 & 5.9135 \\ 0.8660 & 1.7320 & 0 & 1.0146 \\ 1.7320 & 1.7320 & 0 & 11.8271 \\ 1.7320 & 1.7320 & 0 & 2.0292 \\ 0.1689 & 0.3379 & 0.9890 & 1.1539 \\ 0.9986 i & 1.9972 i & 1.0028 i & 1.1699 i \\ 13.3196 & 13.3196 & 13.3756 & 15.6049 \\ 0.1478 & 0.1478 & 0.8655 & 1.0098\end{array}$.


Итак, все решения, кроме двух решений шестой пары, вешественны. Согласно теореме Безу [12] система $n$ однородных полиномиальных уравнений с $(n+1)$ неизвестной, рассматриваемая над полем комплексных чисел, имеет либо бесконечное число решений, либо число решений с учетом их кратности равно произведению степеней уравнений. При этом рассматриваются только ненулевые решения, а пропорциональные считаются за одно. Число вешественных решений системы алгебраических уравнений может быть конечным и бо́льшим произведения степеней уравнений. Пример системы такого рода приведен в [13]. Подобные примеры возможны лишь в случае, когда некоторые из вешественных решений лежат на многообразии комплексных решений положительной размерности. Чтобы убедиться, что все решения нами найдены, достаточно вследствие тонкой теоремы Безу [13] установить изолированность всех шестнадшати найденных комплексных решений. Для этого достаточно проверить неравенство нулю в найденных решениях функциональных определителей:

$$
|d \varphi|=\left|\begin{array}{cccc}
\frac{8}{3} y_{1}-2 y_{2} & \frac{4}{3} y_{2}-2 y_{1} & 0 & 0 \\
4 y_{1}-2 y_{4} & 0 & 0 & \frac{1}{2} y_{4}-2 y_{1} \\
0 & \frac{2}{3} y_{2}-2 y_{3} & \frac{10}{3} y_{3}-2 y_{2} & 0 \\
0 & 0 & \frac{14}{3} y_{3}-2 y_{4} & -2 y_{3}
\end{array}\right| .
$$

Для двух решений из одной пары эти определители очевидным образом совпадают. Приведем их приближенные величины в порядке выписанных пар решений: $19.3,-3.3,-77.3,13.3,-0.7,-4.4,-783.9,0.6$. Откуда можно сделать, во-первых, вывод о том, что все решения системы действительно найдены и, во-вторых, что степень отображения $\varphi$ равна -2 . Вследствие теоремы 6 это доказывает устойчивость рассматриваемого распрямленного шарнирника. Так как степень рычажного отображения равна 0 , то в силу следствия леммы 6 у точки $F(\mathbf{p})$ имеется хотя бы один прообраз, отличный от $\mathbf{p}$. Поскольку $\mathbf{p}$ - ферма, то вследствие теоремы 5 шарнирники, отвечающие этим прообразам, не являются распрямленными. Подсчеты, вьполненные с помощью компьютера, дают пять шарнирников и еще пять зеркально симметричных им относительно оси абсцисс. В следуюшей таблице приведены приближенные значения координат их свободных шарниров (вьписаны четыре знака после запятой без округления):

$$
\begin{array}{cccc}
p_{1} & p_{2} & p_{3} & p_{4} \\
(-2.9282 ; 0.6524) & (-1.9818 ; 0.3291) & (-3.9818 ; 0.3291) & (-0.8452 ; 2.8114) \\
(-2.9653 ; 0.4546) & (-1.9912 ; 0.2283) & (-3.7897 ; 1.1032) & (-1.4011 ;-2.1052) \\
(-2.1255 ; 2.1170) & (-1.7545 ; 1.1884) & (-0.9037 ; 2.9984) & (-1.9031 ;-0.8746) \\
(-1.4646 ; 2.6181) & (-0.4646 ; 2.6181) & (1.3748 ; 1.8331) & (-1.9858 ;-0.3362) \\
(-2.9968 ; 0.1370) & (-1.9968 ; 0.1370) & (-3.9226 ; 0.6766) & (-1.2641 ;-2.3119)
\end{array} .
$$

На рис. $4 \mathrm{~b}$ ) изображен первый из этих шарнирников. Значения функциональных определителей $|d F|$ в точках, отвечающих этим шарнирникам, равны соответственно $30.4603,15.4099,-200.0177,474.2129,-11.1947$, что согласуется с равенством $\operatorname{deg}_{\mathbf{p}} F=2$. 
Из наших подсчетов можно сделать вывод о сушествовании вблизи КШС нашего примера таких кинематических шарнирных схем, которым отвечают $14+10=24$ различных изометричных шарнирника. Это число превосходит очевидную оценку снизу $2^{m}=2^{4}=16$ для числа изометричных шарнирных ферм с четырьмя свободными шарнирами в плоскости в случае изостатической ЗШС. Заметим еше, что, как показывают подсчеты, системы уравнений $\varphi(\mathbf{y})=a$ с различньми правыми частями $a \neq 0$ имеют различное число вешественных решений. Отсюда можно сделать вывод о существовании неустойчивых шарнирников, сколь угодно близких к устойчивому шарнирнику р. Таким образом, в отличие от простейших примеров множество устойчивых шарнирников в данном случае не является открытым.

\section{§6. Необычный пример неустойчивости шарнирника}

Здесь описан пример неустойчивого, но устойчивого по малому изменению длины каждого рычага в отдельности шарнирника, заявленный автором в [11].

Рассмотрим распрямленный шарнирник q (рис. 4c)) с той же ЗШС, что и в предыдущем примере. Свободные его шарниры лежат на прямой $y=0$ в точках $x_{1}=3, x_{3}=4, x_{2}=5, x_{4}=6$. Повторяя для него без каких-либо сушественных изменений подсчеты, проведенные в предыдушем примере, получаем следуюшее задание проекции образуюшего конуса $\mathscr{K}$ на координатное подпространство $\mathscr{R}^{4}$ вдоль касательного в точке q многообразия:

$$
\begin{aligned}
\pi \mathscr{K}= & \left(\frac{5}{3} y_{1}^{2}+\frac{1}{2} y_{2}^{2}-2 y_{1} y_{2}, 2 y_{1}^{2}+\frac{1}{4} y_{4}^{2}-2 y_{1} y_{4}, \frac{3}{4} y_{2}^{2}+\frac{6}{5} y_{3}^{2}-2 y_{2} y_{3},\right. \\
& \left.\frac{7}{5} y_{3}^{2}+\frac{1}{2} y_{4}^{2}-2 y_{3} y_{4}\right) .
\end{aligned}
$$

Далее рассмотрим семнадцать систем из четырех уравнений, левые части которых представляют собой координаты вектора $\pi \mathscr{K}$, а правые части равны столбцам матрицы

$$
\left(\begin{array}{rrrrrrrrrrrrrrrrr}
\frac{2}{3} & -\frac{1}{2} & 0 & 0 & 1 & 0 & 0 & 0 & -\frac{2}{3} & \frac{1}{2} & 0 & 0 & -1 & 0 & 0 & 0 & 5 \\
1 & 0 & 0 & -\frac{3}{4} & 0 & 1 & 0 & 0 & -1 & 0 & 0 & \frac{3}{4} & 0 & -1 & 0 & 0 & 13 \\
0 & -\frac{1}{4} & \frac{1}{5} & 0 & 0 & 0 & 1 & 0 & 0 & \frac{1}{4} & -\frac{1}{5} & 0 & 0 & 0 & -1 & 0 & 12 \\
0 & 0 & \frac{2}{5} & -\frac{1}{2} & 0 & 0 & 0 & 1 & 0 & 0 & -\frac{2}{5} & \frac{1}{2} & 0 & 0 & 0 & -1 & 11
\end{array}\right) .
$$

Первые 16 столбцов этой матрицы представляют собой проекции на $\mathscr{R}^{4}$ вдоль касательного в точке q многообразия векторов единичной длины, лежаших на осях стандартной координатной системы в $\mathscr{R}^{8}$. С помощью пакета Maple можно разыскать у каждой из первых шестнадцати систем точные вешественные решения. Они 
неединственны. Ниже выписаны приближенные значения одного из наборов решений этих первых шестнадцати систем:

$$
\begin{array}{rrrrr}
y_{1} & \multicolumn{1}{c}{y_{2}} & \multicolumn{1}{c}{y_{3}} & \multicolumn{1}{c}{y_{4}} & \multicolumn{1}{c}{|d \varphi|} \\
-1.4379 & -1.2291 & -1.3482 & -1.2195 & -0.2360 \\
-1.2247 & -2.4527 & -1.5862 & -1.4348 & 1.1223 \\
0.0576 & 0.1622 & -0.2752 & 0.3933 & -0.0109 \\
-0.6130 & -1.7266 & -0.9838 & -2.3698 & -0.5744 \\
-0.8398 & -3.2514 & -1.8526 & -5.7348 & -20.8205 \\
1.0702 & 1.2666 & 0.7217 & 0.6528 & 1.0343 \\
0.5006 & 1.4101 & 0.1894 & 0.5865 & -0.7429 \\
-0.4471 & -1.2593 & -0.7175 & -3.0531 & -0.8224 \\
1.4246 & 2.7087 & 2.9710 & 9.1968 & -32.0897 \\
-0.3012 & 0.4273 & -0.1140 & -0.3529 & -0.1936 \\
-0.5508 & -1.5515 & -1.3157 & -3.7617 & -1.0047 \\
0.3743 & 0.4430 & 0.2524 & -0.5326 & 0.1238 \\
1.7322 & 3.4837 & 3.8211 & 11.8282 & -88.5699 \\
-0.7525 & -2.1194 & -1.2077 & -3.7383 & -2.8960 \\
-21.3649 & -25.2854 & -27.6717 & -25.0305 & 1352.8869 \\
-1.9719 & -2.3337 & -1.3298 & -2.3102 & 6.7216
\end{array}
$$

Для всех вьписанных решений значение функционального определителя (10), записанное в последнем столбце, не равно нулю. Это влечет существование малых шариков с центрами на прямых, проходящих через точку $\mathbf{d}=F(\mathbf{q})$ параллельно каждой из стандартных координатных осей в $\mathscr{R}^{8}$, целиком лежаших в касательном конусе $K_{\mathbf{q}}$ рычажного отображения $F$ в точке $\mathbf{q}$. Следовательно, параллельные осям стандартной координатной системы в $\mathscr{R}^{8}$ и проходящие через точку $\mathbf{d}$ прямые лежат внутри конуса $K_{\mathbf{q}}$, что необходимо для устойчивости шарнирника $\mathbf{q}$ по шевелению длины каждого его рычага в отдельности. Достаточность этого условия будет установлена ниже в теореме 8 . С другой стороны, семнадцатая система уравнений, вектор правой части которой подобран с помощью генератора случайных чисел, не имеет вешественных решений. В этом автор убедился так. С помощью пакета Maple были найдены в точном виде все 8 с точностью до знака пар ее решений, оказавшиеся комплексными. Приведем приближенные значения переменной $y_{3}$ для каждой из этих 8 пар решений:

$$
\begin{array}{cc}
-1.7527-2.7267 i, & 10.3444 i, \\
-0.5469-5.4382 i, & 0.1928-3.0414 i, \\
-0.1928-3.0414 i, & 0.5469-5.4382 i, \\
-79.2033 i, & 1.7527-2.7267 i
\end{array}
$$

Вычисляя функциональные определители $|d \varphi|$ в отвечающих всем этим решениям точках, убеждаемся в их неравенстве нулю. Их значения таковы:

$$
\begin{array}{cc}
-390964.71+670393.56 i, & -3261.45, \\
-8.24+235.91 i, & 113.59+565.21 i, \\
113.59-565.21 i, & -8.24-235.91 i, \\
90145.57, & -390964.71-670393.56 i .
\end{array}
$$


В силу тонкой теоремы Безу других решений семнадцатая система не имеет. Итак, мы нашли луч, не лежаший в касательном конусе $K_{\mathbf{q}}$. Необходимое условие устойчивости шарнирника $\mathbf{q}$ не выполнено.

Докажем наконец теорему 8 , необходимую для обоснования этого примера.

ТЕОРема 8. Пусть система уравнений $\varphi(\mathbf{y})=w$ имеет решение $\mathbf{y}_{0}$, для которого $\operatorname{det} d \varphi\left(\mathbf{y}_{0}\right) \neq 0$. Тогда система $(5)$

$$
\left\{\begin{array}{l}
\varphi(\mathbf{y})+\phi(\boldsymbol{\xi})=s w \\
\mathscr{A} \boldsymbol{\xi}+\vartheta(\mathbf{y})+\theta(\boldsymbol{\xi})=s t
\end{array}\right.
$$

имеет для произвольного фиксированного вектора $t \in T$ и всех достаточно малых вещественных $s>0$ решение $(\boldsymbol{\xi}, \mathbf{y})$, близкое $к$ нулевому вектору.

ДокАЗАТЕЛЬСТво проведем методом сжимающих отображений. В качестве начальных приближений возьмем

$$
\mathbf{y}^{[0]}=\mathbf{y}^{[0]}(s)=\varphi^{-1}(s w), \quad \boldsymbol{\xi}^{[0]}=\boldsymbol{\xi}^{[0]}(s)=\mathscr{A}^{-1}\left(s t-\vartheta\left(\mathbf{y}^{[0]}(s)\right)\right) .
$$

Заметим, что начальные приближения зависят от параметра $s$ следующим образом:

$$
\mathbf{y}^{[0]}(s)=\sqrt{s} \mathbf{y}^{[0]}(1)=\sqrt{s} \mathbf{y}_{0}, \quad \boldsymbol{\xi}^{[0]}(s)=s \boldsymbol{\xi}^{[0]}(1)=s \mathscr{A}^{-1}\left(t-\vartheta\left(\mathbf{y}_{0}\right)\right) .
$$

Последующие приближения будем находить по формулам

$$
\mathbf{y}^{[i]}=\varphi^{-1}\left(s w-\phi\left(\boldsymbol{\xi}^{[i-1]}\right)\right), \quad \boldsymbol{\xi}^{[i]}=\mathscr{A}^{-1}\left(s t-\vartheta\left(\mathbf{y}^{[i]}\right)-\theta\left(\boldsymbol{\xi}^{[i-1]}\right)\right) .
$$

Подставляя выражение $\mathbf{y}^{[i]}$ из первой из этих формул во вторую, получаем формулу процесса последовательных приближений, включающую только переменную $\boldsymbol{\xi}$ :

$$
\boldsymbol{\xi}^{[i]}=\mathscr{A}^{-1}\left(s t-\vartheta\left(\varphi^{-1}\left(s w-\phi\left(\boldsymbol{\xi}^{[i-1]}\right)\right)\right)-\theta\left(\boldsymbol{\xi}^{[i-1]}\right)\right) .
$$

Если мы докажем при произвольном $t$ и всех достаточно малых $s$ существование близкого к нулевому решения $\boldsymbol{\xi}$ системы уравнений

$$
\boldsymbol{\xi}=\mathscr{A}^{-1}\left(s t-\vartheta\left(\varphi^{-1}(s w-\phi(\boldsymbol{\xi}))\right)-\theta(\boldsymbol{\xi})\right),
$$

то тем самым будет доказано сушествование близкого к нулевому решения $(\boldsymbol{\xi}, \mathbf{y})$, $\mathbf{y}=\varphi^{-1}(s w-\phi(\boldsymbol{\xi}))$, и для системы (5) при тех же условиях. Действительно, будем считать все точки, получаюшиеся в процессе последовательных приближений, удовлетворяюшими для некоторых $p>0, q>0$ условиям $|\boldsymbol{\xi}|<p s$, $|\mathbf{y}|=\left|\varphi^{-1}(s w-\phi(\boldsymbol{\xi}))\right|<q \sqrt{s}$. (В дальнейшем мы увидим, что это предположение оправдано при достаточно малых $s$.) В качестве отображения $\varphi^{-1}$ мы берем непрерывную ветвь отображения, обратного к $\varphi$, для которой $\varphi^{-1}(w)=\mathbf{y}_{0}$. В самом деле, поскольку $\operatorname{det} d \varphi\left(\mathbf{y}_{0}\right) \neq 0$ и отображение $\varphi$ гладкое, то по теореме об обратном отображении отображение $\varphi^{-1}$ определено в шаре некоторого радиуса $r>0$ с центром в точке $w$. А вследствие однородности квадратичного отображения $\varphi$ оно определено и в шаре радиуса $s r$ с центром $s w$, причем $\varphi^{-1}(s w)=\sqrt{s} \mathbf{y}_{0}$. 
Для однородного квадратичного отображения $\phi(\boldsymbol{\xi})$ существует такая постоянная $l$, что $\phi(\boldsymbol{\xi}) \leqslant l|\boldsymbol{\xi}|^{2}$. Следовательно, для корректности наших итераций должно выполняться условие $l|\boldsymbol{\xi}|^{2} \leqslant l(p s)^{2} \leqslant r s$. Что имеет место в случае $s \leqslant r /\left(l p^{2}\right)=B$.

Для доказательства сушествования решения системы уравнений (11) методом сжимающих отображений требуется установить, что отображение $F_{s}$, которое задают правые части этой системы, является сжимающим при всех достаточно малых $s$, а образ шара $B_{s}:\left|\boldsymbol{\xi}-\boldsymbol{\xi}_{s}^{[0]}\right|<\rho s$ при некотором $0<\rho<r$ лежит в этом шаре.

Пусть $f(x, y)$ - симметрическое билинейное отображение, полярное однородному квадратичному отображению $f(x, x)=F(x)$ (его координатные функции суть билинейные формы, полярные квадратичным формам, за дающим отображение $F$ ). Тогда справедливы следуюшие соотношения:

$$
\begin{aligned}
F(y) & =f(y, y)=f(x+(y-x), x+(y-x)) \\
& =f(x, x)+2 f(x, y-x)+f(y-x, y-x), \\
F(y)-F(x) & =2 f(x, y-x)+f(y-x, y-x) .
\end{aligned}
$$

Откуда следует

$$
\begin{aligned}
|F(y)-F(x)| & =|2 f(x, y-x)+f(y-x, y-x)| \leqslant|2 f(x, y-x)|+|f(y-x, y-x)| \\
& \leqslant b\left(2|x||y-x|+|y-x|^{2}\right)=b|y-x|(2|x|+|y-x|) .
\end{aligned}
$$

Здесь в качестве $b$ можно взять число $n^{2} m M$, где $n$ - размерность пространства прообраза, $m$ - размерность пространства образа, а $M$ - наибольшая из абсолютных величин коэффициентов билинейных форм, осуществляюших отображение $f(x, y)$.

С учетом (12), считая выполненным условие $s \leqslant B$, имеем

$$
\begin{aligned}
& \left|F_{s}\left(\boldsymbol{\xi}_{1}\right)-F_{s}\left(\boldsymbol{\xi}_{2}\right)\right|=\left|\mathscr{A}^{-1}\left(s t-\vartheta\left(\mathbf{y}_{1}\right)-\theta\left(\boldsymbol{\xi}_{1}\right)\right)-\mathscr{A}^{-1}\left(s t-\vartheta\left(\mathbf{y}_{2}\right)-\theta\left(\boldsymbol{\xi}_{2}\right)\right)\right| \\
& \quad \leqslant\left|\mathscr{A}^{-1}\left(\vartheta\left(\mathbf{y}_{2}\right)-\vartheta\left(\mathbf{y}_{1}\right)\right)\right|+\left|\mathscr{A}^{-1}\left(\theta\left(\boldsymbol{\xi}_{2}\right)-\theta\left(\boldsymbol{\xi}_{1}\right)\right)\right| \\
& \quad \leqslant a\left(\left|\vartheta\left(\mathbf{y}_{2}\right)-\vartheta\left(\mathbf{y}_{1}\right)\right|+\left|\theta\left(\boldsymbol{\xi}_{2}\right)-\theta\left(\boldsymbol{\xi}_{1}\right)\right|\right) \\
& \quad \leqslant a\left(b_{\vartheta}\left|\mathbf{y}_{2}-\mathbf{y}_{1}\right|\left(2\left|\mathbf{y}_{1}\right|+\left|\mathbf{y}_{2}-\mathbf{y}_{1}\right|\right)+b_{\theta}\left|\boldsymbol{\xi}_{2}-\boldsymbol{\xi}_{1}\right|\left(2\left|\boldsymbol{\xi}_{1}\right|+\left|\boldsymbol{\xi}_{2}-\boldsymbol{\xi}_{1}\right|\right)\right)
\end{aligned}
$$

причем постоянная $а$ зависит лишш от отображения $\mathscr{A}^{-1}$, а $b_{\vartheta}$ и $b_{\theta}$ связаны с отображениями $\vartheta$ и $\theta$ так же, как $b$ c $F$. Далее, можно записать, используя формулу конечных приращений Лагранжа для функции $\varphi_{i}^{-1}$, представляющей собой координатную функцию отображения $\varphi^{-1}$,

$$
\begin{aligned}
& \varphi_{i}^{-1}\left(s w-\phi\left(\boldsymbol{\xi}_{2}\right)\right)-\varphi_{i}^{-1}\left(s w-\phi\left(\boldsymbol{\xi}_{1}\right)\right) \\
& \quad=\left(\operatorname{grad} \varphi_{i}^{-1}\left(s w-\theta_{i} \phi\left(\boldsymbol{\xi}_{1}\right)+\left(\theta_{i}-1\right) \phi\left(\boldsymbol{\xi}_{2}\right)\right), \phi\left(\boldsymbol{\xi}_{1}\right)-\phi\left(\boldsymbol{\xi}_{2}\right)\right),
\end{aligned}
$$

где $0<\theta_{i}<1$. Заметим, что поскольку функциональная матрица отображения $\varphi$ обладает свойством $d \varphi(\sqrt{s} \mathbf{y})=\sqrt{s} d \varphi(\mathbf{y})$, то функциональная матрица обратного отображения, являясь обратной к ней, обладает свойством $d \varphi^{-1}(s w)=$ 
$(1 / \sqrt{s}) d \varphi^{-1}(w)$. В силу гладкости отображения $\varphi^{-1}$ вблизи точки $s w$, следующей из теоремы об обратном отображении, существуют такие положительные постоянные $c$ и $C$, что для всех $0<s<C$ будет выполнено

$$
\left|\mathbf{y}_{2}-\mathbf{y}_{1}\right|=\left|\varphi^{-1}\left(s w-\phi\left(\boldsymbol{\xi}_{2}\right)\right)-\varphi^{-1}\left(s w-\phi\left(\boldsymbol{\xi}_{1}\right)\right)\right| \leqslant \frac{c}{\sqrt{s}}\left|\phi\left(\boldsymbol{\xi}_{1}\right)-\phi\left(\boldsymbol{\xi}_{2}\right)\right| .
$$

Теперь с учетом (14) и условий $\left|\boldsymbol{\xi}_{i}\right|<p s,\left|\mathbf{y}_{i}\right|<q \sqrt{s}, i=1,2$, получаем из (13)

$$
\begin{aligned}
& \left|F_{s}\left(\boldsymbol{\xi}_{1}\right)-F_{s}\left(\boldsymbol{\xi}_{2}\right)\right| \leqslant a\left(b_{\vartheta} \frac{c}{\sqrt{s}}\left|\phi\left(\boldsymbol{\xi}_{1}\right)-\phi\left(\boldsymbol{\xi}_{2}\right)\right| 4 q \sqrt{s}+b_{\theta}\left|\boldsymbol{\xi}_{2}-\boldsymbol{\xi}_{1}\right|\left(2\left|\boldsymbol{\xi}_{1}\right|+\left|\boldsymbol{\xi}_{2}-\boldsymbol{\xi}_{1}\right|\right)\right) \\
& \quad \leqslant a\left(4 b_{\vartheta} c q b_{\phi}\left|\boldsymbol{\xi}_{2}-\boldsymbol{\xi}_{1}\right|\left(2\left|\boldsymbol{\xi}_{1}\right|+\left|\boldsymbol{\xi}_{2}-\boldsymbol{\xi}_{1}\right|\right)+b_{\theta}\left|\boldsymbol{\xi}_{2}-\boldsymbol{\xi}_{1}\right|\left(2\left|\boldsymbol{\xi}_{1}\right|+\left|\boldsymbol{\xi}_{2}-\boldsymbol{\xi}_{1}\right|\right)\right) \\
& \leqslant a\left(4 b_{\vartheta} c q b_{\phi}+b_{\theta}\right)\left|\boldsymbol{\xi}_{2}-\boldsymbol{\xi}_{1}\right|\left(2\left|\boldsymbol{\xi}_{1}\right|+\left|\boldsymbol{\xi}_{2}-\boldsymbol{\xi}_{1}\right|\right) \leqslant\left(4 b_{\vartheta} c q b_{\phi}+b_{\theta}\right) 4 a p s\left|\boldsymbol{\xi}_{2}-\boldsymbol{\xi}_{1}\right| .
\end{aligned}
$$

Таким образом, отображение $F_{s}$ является сжимающим для всех $s$, меньших

$$
\frac{k}{4 a p\left(4 b_{\vartheta} c q b_{\phi}+b_{\theta}\right)}=D(k), \quad 0<k<1
$$

Пусть теперь $\left|\boldsymbol{\xi}-\boldsymbol{\xi}^{[0]}(s)\right| \leqslant \rho s$, тогда имеем

$$
\left|F_{s}(\boldsymbol{\xi})-\boldsymbol{\xi}^{[0]}(s)\right|=\left|F_{s}(\boldsymbol{\xi})-F_{s}(0)\right| \leqslant\left(4 b_{\vartheta} c q b_{\phi}+b_{\theta}\right) 4 a p s|\boldsymbol{\xi}| \leqslant\left(4 b_{\vartheta} c q b_{\phi}+b_{\theta}\right) 4 a p^{2} s^{2} .
$$

Левая часть этого неравенства не превосходит $\rho s$, если

$$
s \leqslant \frac{\rho}{\left(4 b_{\vartheta} c q b_{\phi}+b_{\theta}\right) 4 a p^{2}}=E(\rho) .
$$

Итак, для $s$, меньших, скажем, $\min \left\{B, C, D\left(\frac{1}{2}\right), E\left(\frac{r}{2}\right)\right\}$, условия метода сжимающих отображений выполнены и, следовательно, существует решение $(\boldsymbol{\xi}, \mathbf{y})$ системы уравнений $(5)$ с $|\boldsymbol{\xi}| \leqslant p s,|\mathbf{y}| \leqslant q \sqrt{s}$. Причем для выбранной ветви отображения $\varphi^{-1}$ это решение единственно.

\section{Список литературы}

1. Connelly R. Rigidity // Handbook of convex geometry / ed. P. Gruber et al. V. A. Amsterdam: North-Holland, 1993. P. 223-271.

2. White N., Whiteley $W$. The algebraic geometry of stresses in frameworks // SIAM J. Algebraic Discrete Methods. 1983. V. 4. № 4. P. 481-511.

3. Ковалев М. Д. Устойчивость шарнирников, шарнирных устройств и схем // Итоги науки и техники. Совр. матем. и ее прилож. Т. 68. (Труды международной конференции, посвященной 90-летию со дня рождения Л. С. Понтрягина. Т. 7. Топология и геометрия.) М.: ВИНИТИ, 1999. С. 65-86.

4. Ковалев М. Д. Геометрическая теория шарнирных устройств // Изв. РАН. Сер. матем. 1994. Т. 58. №1. С. $45-70$.

5. Ковалев М. Д. Квадратичные и рычажные отображения // Труды МИАН. Дискретная геометрия, геометрия чисел и приложения. 2002. Т. 239. С. 195-214.

6. Аграчев А.А., Гамкрелидзе Р. В. Квадратичные отображения и гладкие функции: эйлеровы характеристики множеств уровня // Итоги науки и техники. Совр. проблемы матем. Т. 35. М.: ВИНИТИ, 1989. С. 179-239. 
7. Ковалев М. Д. О восстановимости шарнирников по внутренним напряжениям // Изв. РАН. Сер. матем. 1997. Т. 61. № 4. С. 37-66.

8. Берже М. Геометрия. Т. 1. М.: Мир, 1984.

9. Дубровин В.А., Новиков С. П., Фоменко А. Т. Современная геометрия. М.: Наука, 1979.

10. Арнольд В.И., Варченко А.Н., Гусейн-Заде С. М. Особенности дифференцируемых отображений. М.: Наука, 1982.

11. Kovalev $M$. Local properties of the rigidity mapping // Convex and discrete geometry. Abstracts. Bydgoszcz. Poland: Institut Matematyki i Fizyki ATR, 1998. P. 40.

12. Шафаревич И. Р. Основы алгебраической геометрии. Т. 1. М.: Наука, 1988.

13. Фултон У. Теория пересечений. М.: Мир, 1989.

Московский государственнњй

технический университет

им. Н.Э. Баумана
Поступила в редакцию

08.02 .2002 и 24.03 .2003 